\title{
THE REALIZABILITY OF CURVES IN A TROPICAL PLANE
}

\author{
ANDREAS GATHMANN, KIRSTEN SCHMITZ, AND ANNA LENA WINSTEL
}

\begin{abstract}
Let $E$ be a plane in an algebraic torus over an algebraically closed field. Given a balanced 1-dimensional fan $C$ in the tropicalization of $E$, i. e. in the Bergman fan of the corresponding matroid, we give a complete algorithmic answer to the question whether or not $C$ can be realized as the tropicalization of an algebraic curve contained in $E$. Moreover, in the case of realizability the algorithm also determines the dimension of the moduli space of all algebraic curves in $E$ tropicalizing to $C$, a concrete simple example of such a curve, and whether $C$ can also be realized by an irreducible algebraic curve in $E$. In the first important case when $E$ is a general plane in a 3-dimensional torus we also use our algorithm to prove some general criteria for $C$ that imply its realizability resp. non-realizability. They include and generalize the main known obstructions by Brugallé-Shaw and Bogart-Katz coming from tropical intersection theory.
\end{abstract}

\section{INTRODUCTION}

Tropical geometry is a branch of mathematics that establishes a deep connection between algebraic geometry and combinatorics. For example, given a $k$-dimensional subvariety $Y$ of an $n$-dimensional algebraic torus $X$ over an algebraically closed field, the process of tropicalization assigns to it a purely $k$-dimensional polyhedral fan $\operatorname{trop}(Y)$ in an $n$-dimensional real vector space, together with a positive integer multiplicity assigned to each facet [Spe05]. Although this fan is in a certain sense a simpler object than the original variety $Y$, it still carries much information about $Y$. It is therefore the idea of tropical geometry to study these fans by combinatorial methods, and then transfer the results back to algebraic geometry.

In order for this strategy to work efficiently it is of course essential to know which fans can actually occur as tropicalizations of algebraic varieties - this is usually called the realization problem or tropical inverse problem. An important well-known necessary condition for a fan together with given multiplicities on the facets to be realizable as the tropicalization of an algebraic variety is the so-called balancing condition, certain linear relations among the multiplicities of the adjacent cells of each codimension-1 cone [Spe05, Section 2.5]. In the case of fans of dimension or codimension 1 this condition is also sufficient for realizability by an algebraic curve resp. hypersurface [NS06, Spe05, Spe07, Mik02], but for intermediate dimensions no such general statements are known so far.

Rather than considering varieties of intermediate dimension, we will restrict ourselves in this paper to the case of curves and study a relative version of the realization problem:

Key words and phrases. Tropical geometry, tropicalization, tropical realizability.

2010 Mathematics Subject Classification: 14T05.

Kirsten Schmitz has been supported by the DFG grant Ga 636/3. 
let $E$ be a fixed plane in $X$, i. e. a 2-dimensional subvariety of an algebraic torus defined by linear equations. Its tropicalization $\operatorname{trop}(E)$ is the 2-dimensional Bergman fan of the corresponding matroid [Stu02, AK06]. Given a balanced 1-dimensional fan $C$ with rays in the support of $\operatorname{trop}(E)$ - in the following we will call this a tropical curve in trop $(E)$ - the relative realization problem then is to decide whether there is a (maybe reducible) algebraic curve $Y$ in $E$ that tropicalizes to $C$. Results in this direction are useful if one wants to use tropical methods to analyze the geometry of algebraic curves in (a toric compactification of) $E$, e. g. for setting up moduli spaces of such curves or studying the cone of effective curve classes.

The first important example of this situation is that of a general plane $E$ in a 3 -dimensional torus $X$. In this case (the support of) $\operatorname{trop}(E)$ will be denoted by $L_{2}^{3}$; it is the union of all cones generated by two of the classes $\left[e_{0}\right], \ldots,\left[e_{3}\right]$ of the unit vectors in $\mathbb{R}^{4} /\langle\mathbf{1}\rangle$, where $\mathbf{1}=(1,1,1,1)$. The picture on the right shows this space, together with an example of a tropical curve $C$ in it. Its rays all have multiplicity 1 and are spanned by the vectors (in homogeneous coordinates)

$$
[0,3,1,0],[0,0,1,3],[2,0,1,0] \text {, and }[1,0,0,0] \text {. }
$$

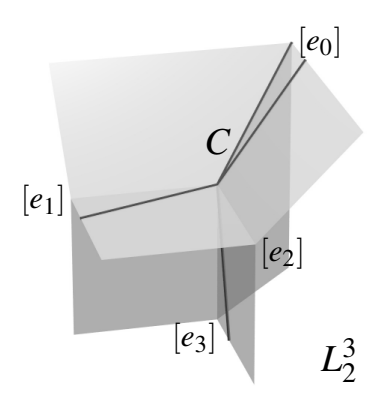

Note that the balancing condition in this case just means that these four vectors add up to 0 in $\mathbb{R}^{4} /\langle\mathbf{1}\rangle$. As the above representatives (normalized so that their minimal coordinate is 0 ) sum up to $(3,3,3,3)$ any algebraic curve realizing $C$ must have degree 3 (see Example 2.10 and Lemma 4.5). We are thus asking if there is a cubic curve in $E$ tropicalizing to $C$.

Several necessary conditions for this relative realizability have been known so far, all of them coming from the comparison of tropical and classical intersection theory. The strongest obstruction seems to be that of Brugallé and Shaw, stating that an irreducible tropical curve $C$ in $\operatorname{trop}(E)$ cannot be realizable if it has a negative intersection product with another realizable irreducible tropical curve $D \neq C$ [BS11, Corollary 3.10], e. g. if $D$ is one of the three straight lines contained in $L_{2}^{3}$. They also prove obstructions coming from the adjunction formula and intersection with the Hessian [BS11, Sections 4 and 5]. In addition, Bogart and Katz have shown that a tropical curve in $E$ contained in a classical hyperplane can only be realizable if it contains a classical line or is a multiple of the tropical intersection product of $E$ with this hyperplane [BK11, Proposition 1.3]. However, none of these criteria are also sufficient for realizability. They all fail to detect some of the non-realizable curves - e.g. the curve $C$ in $L_{2}^{3}$ in the picture above, which actually turns out to be non-realizable by an algebraic curve in $E$ (see Proposition 5.15 and Example 5.23).

In this paper we will take a different approach to the relative realization problem. It is of an algorithmic nature, and thus first of all leads to an efficient way to decide for any given tropical curve $C$ in $\operatorname{trop}(E)$ whether or not it is realizable by an algebraic curve in $E$. After recalling the basic tropical background in section 2, we then show in sections 3 and 4 that checking whether the tropicalization of an algebraic curve is equal to $C$ is equivalent to checking that the projections of the curve to the various coordinate planes tropicalize to the corresponding projections of $C$ to $\mathbb{R}^{3} /\langle\mathbf{1}\rangle$. As these checks are now in the plane, they can 
easily be performed explicitly by comparing Newton polytopes. The resulting Algorithm 4.15 to decide for relative realizability is also available for download as a Singular library [DGPS, Win12]. It can distinguish between realizability by a reducible and by an irreducible curve, compute the dimension of the space of algebraic curves tropicalizing to $C$ (which in fact is an open subset of a linear space), and provide an explicit easy example of such an algebraic curve in case of realizability. The computations can be performed for ground fields of any characteristic, and in fact the results will in general depend on this choice (see Example 5.24).

From the numeric results of these computations it seems unlikely that there is a general easy rule to decide for realizability in any given case. However, by a systematic study of the algorithm we prove some criteria in section 5 that imply realizability resp. non-realizability in many cases of interest. In the case of $L_{2}^{3}$ they include and generalize the main previously known obstructions by Brugallé-Shaw and Bogart-Katz mentioned above, thus putting them into a common framework with a unified idea of proof (see Propositions 5.10 and 5.21). In addition, our criteria show that every tropical curve in $\operatorname{trop}(E)$ can be realized by an algebraic cycle in $E$, i. e. by a formal $\mathbb{Z}$-linear combination of algebraic curves in $E$ (see Proposition 5.3).

One example of a new obstruction to realizability in the case of tropical curves in $L_{2}^{3}$ is shown in the picture on the right: a tropical curve that is completely contained in the shaded area cannot be realizable by an algebraic curve in $E$ if its multiplicity on the ray $\left[e_{0}\right]$ is $1-$ regardless of the characteristic of the ground field (see Proposition 5.15). This shows e.g. the nonrealizability of the example curve that we had considered in the picture above.

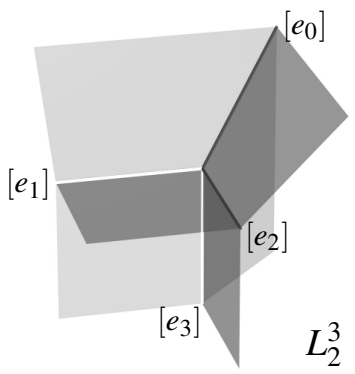

The following numbers may be useful to get a feeling for the numerical complexity of the problem: there are (up to coordinate permutations) 182 tropical curves of degree 3 and 2122 curves of degree 4 in $L_{2}^{3}$. In characteristic zero, 17 of the degree 3-curves and 138 of the degree- 4 curves are not realizable. Checking the realizability of all these curves takes less than one minute on a standard PC. In degree 3 our general criteria suffice to find all nonrealizable curves, whereas 21 of the 138 non-realizable curves remain undetected by these obstructions in degree 4 (see Example 5.23).

It should be noted that the methods of this paper are quite general and can also be applied e. g. to the "non-constant coefficient case", i. e. to the question which 1-dimensional balanced polyhedral complexes in $\operatorname{trop}(E)$ can be realized as the tropicalization of an algebraic curve in $E$ over a non-Archimedean valued field. Work in this direction is in progress.

\section{Tropical Geometry}

We will start by recalling the basic combinatorial concepts from tropical geometry used in this paper. More details can be found e.g. in [AR10]. 
Notation 2.1 (Tropical cycles). Let $n \in \mathbb{N}$, let $\Lambda$ be a lattice of rank $n$, and let $V=\Lambda \otimes_{\mathbb{Z}} \mathbb{R}$ be the corresponding real vector space. By a cone $\sigma$ in $V$ we will always mean a rational polyhedral cone. Let $V_{\sigma} \subset V$ be the vector space spanned by $\sigma$, and $\Lambda_{\sigma}:=V_{\sigma} \cap \Lambda$. If the cone $\tau$ is a face of $\sigma$ of codimension 1 , we denote by $u_{\sigma / \tau} \in \Lambda_{\sigma} / \Lambda_{\tau}$ the primitive normal vector of $\sigma$ modulo $\tau$. In the case $\operatorname{dim} \sigma=1$ we write $u_{\sigma /\{0\}} \in \Lambda_{\sigma} \subset \Lambda$ also as $u_{\sigma}$.

For $r \in \mathbb{N}$ an $r$-dimensional (tropical) cycle or $r$-cycle in $V$ is a pure $r$-dimensional fan $C$ of cones in $V$ as above, together with a multiplicity $m_{C}(\sigma) \in \mathbb{Z}$ for each maximal cone $\sigma \in C$, and such that the balancing condition

$$
\sum_{\sigma>\tau} m_{C}(\sigma) u_{\sigma / \tau}=0 \quad \in V / V_{\tau}
$$

holds for each $(r-1)$-dimensional cone $\tau \in C$ (where the sum is taken over all maximal cones $\sigma$ containing $\tau$ as a face). If there is no risk of confusion, we will also write $m(\sigma)$ instead of $m_{C}(\sigma)$. A tropical cycle with only non-negative multiplicities will be called a tropical variety, resp. a tropical curve if $r=1$.

The support $|C| \subset V$ of a tropical cycle $C$ is the union of its maximal cones that have non-zero multiplicity. If $D$ is another tropical cycle in $V$ with $|D| \subset|C|$ we say that $D$ is contained in $C$, and also write this as $D \subset C$ by abuse of notation. The abelian group of all $k$-dimensional cycles contained in $C$, modulo refinements as in [AR10, Definition 2.12], will be denoted by $Z_{k}^{\text {trop }}(C)$.

Construction 2.2 (Intersection products). A rational function on a $k$-dimensional cycle $C$ is a continuous piecewise integer linear function $\varphi:|C| \rightarrow \mathbb{R}$, where we will assume the fan structure of $C$ to be fine enough so that $\varphi$ is linear on each cone $\sigma$, see [AR10, Definition 3.1]. This linear function, extended uniquely to $V_{\sigma}$, will be denoted $\varphi_{\sigma}$. We then define the intersection product $\varphi \cdot C \in Z_{k-1}^{\text {trop }}(C)$ to be the cycle whose maximal cones are the $(k-1)$ dimensional cones $\tau$ of $C$ with multiplicities

$$
m_{\varphi \cdot C}(\tau)=\varphi_{\tau}\left(\sum_{\sigma>\tau} m_{C}(\sigma) v_{\sigma / \tau}\right)-\sum_{\sigma>\tau} m_{C}(\sigma) \varphi_{\sigma}\left(v_{\sigma / \tau}\right),
$$

where the sum is taken over all $k$-dimensional cones $\sigma$ in $C$ containing $\tau$ as a face, and the vectors $v_{\sigma / \tau}$ are arbitrary representatives of $u_{\sigma / \tau}$ [AR10, Definition 3.4]. Its support is contained in the locus of points at which $\varphi$ is not locally linear.

If $\varphi \cdot V=D$ we say that the rational function $\varphi$ cuts out $D$, and write the intersection product $\varphi \cdot C$ also as $D \cdot C$. This intersection product of a codimension-1 cycle $D$ with $C$ is well-defined (i.e. independent of the rational function cutting out $D$ ), and satisfies the expected properties as e.g. commutativity if $C$ can also be cut out by a rational function [AR10, Section 9].

Construction 2.3 (Push-forward of cycles). Let $f: \Lambda \rightarrow \Lambda^{\prime}$ be a linear map of lattices. By abuse of notation, the corresponding linear map of vector spaces $V=\Lambda \otimes_{\mathbb{Z}} \mathbb{R} \rightarrow V^{\prime}=$ $\Lambda^{\prime} \otimes_{\mathbb{Z}} \mathbb{R}$ will also be denoted by $f$. For $C \in Z_{k}^{\text {trop }}(V)$ there is then an associated pushforward cycle $f_{*}(C) \in Z_{k}^{\text {trop }}\left(V^{\prime}\right)$ obtained as follows: subdivide $C$ so that the collection of 
cones $\{f(\sigma): \sigma \in C\}$ is a fan in $V^{\prime}$, and associate to each such image cone $\tau$ of dimension $k$ the multiplicity

$$
m_{f_{*} C}(\tau)=\sum_{\sigma: f(\sigma)=\tau} m_{C}(\sigma) \cdot\left[\Lambda_{\tau}^{\prime}: f\left(\Lambda_{\sigma}\right)\right] .
$$

This way one indeed obtains a balanced cycle, and the corresponding push-forward map $f_{*}: Z_{k}^{\text {trop }}(V) \rightarrow Z_{k}^{\text {trop }}\left(V^{\prime}\right)$ is a homomorphism that satisfies all expected properties as e. $\mathrm{g}$. the projection formula [AR10, Section 4].

Convention 2.4 (Homogeneous coordinates). In the following, we will always work with real vector spaces that have fixed homogeneous coordinates, i.e. we have $V=\mathbb{R}^{N} /\langle\mathbf{1}\rangle$ for a finite index set $N$, where $\mathbf{1}$ denotes the vector all of whose coordinates are equal to 1 . It is then always understood that the underlying lattice is $\mathbb{Z}^{N} /\langle\mathbf{1}\rangle$. The class of a vector $v \in \mathbb{R}^{N}$ in $\mathbb{R}^{N} /\langle\mathbf{1}\rangle$ will be written $[v]$; for $i \in N$ the unit vector in $\mathbb{Z}^{N}$ with entry 1 in the coordinate $i$ is denoted by $e_{i}$. Often we will just have $N=\{0, \ldots, n\}$, in which case we write $V$ as $\mathbb{R}^{n+1} /\langle\mathbf{1}\rangle$ with lattice $\mathbb{Z}^{n+1} /\langle\mathbf{1}\rangle$.

The reason for this choice is that these are the natural ambient spaces for matroid fans tropical varieties that will be central in this paper as they occur as tropicalizations of linear spaces [AK06, Theorem 1]. Let us now introduce these matroid fans from a combinatorial point of view. Details on matroid theory can be found in [Ox192].

Construction 2.5 (Matroid fans). Let $M$ be a loop-free matroid on a finite ground set $N$. By a chain of flats (of length $m$ ) in $M$ we will mean a sequence $\mathscr{F}=\left(F_{1}, \ldots, F_{m}\right)$ of flats of $M$ with

$$
\emptyset \subsetneq F_{1} \subsetneq F_{2} \subsetneq \cdots \subsetneq F_{m} \subsetneq N
$$

For such a chain of flats let $\sigma_{\mathscr{F}} \subset \mathbb{R}^{N} /\langle\mathbf{1}\rangle$ be the $m$-dimensional simplicial cone generated by the classes of the vectors $v_{F_{1}}, \ldots, v_{F_{m}}$, where $v_{F} \in \mathbb{R}^{N}$ for a flat $F$ denotes the vector with entries 1 in the coordinates of $F$, and 0 otherwise. One can show that the collection of all cones $\sigma_{\mathscr{F}}$ corresponding to chains of flats in $M$, with multiplicity 1 assigned to each maximal cone, is a tropical variety of dimension equal to the rank of $M$ minus 1 [Fra12, Proposition 3.1.10]. It is called the matroid fan or Bergman fan associated to $M$ and denoted by $B(M)$.

Example 2.6 (General linear spaces $\left.L_{k}^{n}\right)$. Let $n, k \in \mathbb{N}$ with $k \leq n$, and let $M$ be the uniform matroid of rank $k+1$ on $N=\{0, \ldots, n\}$. Then the matroid fan $B(M)$ consists of the cones spanned by the vectors $\left[v_{F_{1}}\right], \ldots,\left[v_{F_{m}}\right]$ for all sequences $\emptyset \subsetneq F_{1} \subsetneq \cdots \subsetneq F_{m} \subsetneq N$ with $\left|F_{m}\right| \leq k$. We denote it by $L_{k}^{n}$; the picture on the right shows the case of $L_{2}^{3}$ in $\mathbb{R}^{4} /\langle\mathbf{1}\rangle$ (see also Example 3.5).

In the following we will consider tropical cycles only up to refinements. Hence, we will often draw $L_{2}^{3}$ without the subdivision induced by the rank-2 flats.

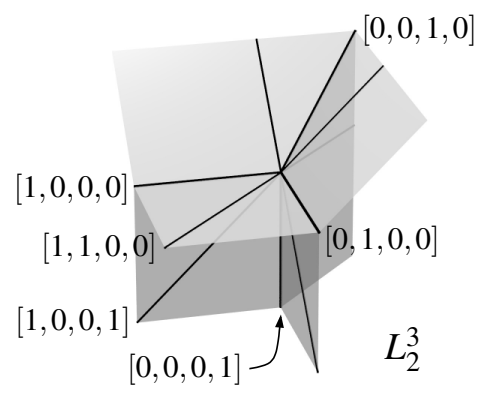

Our main tropical objects in this paper will be tropical curves in matroid fans. So let us now introduce some convenient notations to deal with such curves. 
Notation 2.7 (Description of a curve $C$ with the set $P(C)$ ). For a tropical curve $C$ in $\mathbb{R}^{N} /\langle\mathbf{1}\rangle$ we will always assume that it is subdivided so that the origin is a cone of $C$. If $\sigma_{1}, \ldots, \sigma_{k}$ are the 1-dimensional cones of $C$, we set

$$
P(C):=\left\{m\left(\sigma_{1}\right) v_{1}, \ldots, m\left(\sigma_{k}\right) v_{k}\right\} \quad \subset \mathbb{Z}^{N},
$$

where $v_{i} \in \mathbb{Z}^{N}$ for $i=1, \ldots, k$ is the unique representative of the primitive normal vector $u_{\sigma_{i}} \in \mathbb{Z}^{n} /\langle\mathbf{1}\rangle$ such that the minimum over all its coordinates is 0 . For $v \in \mathbb{Z}^{N}$ denote by $\operatorname{gcd}(v)$ the (non-negative) greatest common divisor of the coordinates of $v$. Then $\operatorname{gcd}\left(v_{i}\right)=$ 1 , and so for all $i$ we have $\operatorname{gcd}\left(m\left(\sigma_{i}\right) v_{i}\right)=m\left(\sigma_{i}\right)$ and $\left[m\left(\sigma_{i}\right) v_{i}\right] \in \sigma_{i}$. This means that the set $P(C)$ allows to reconstruct the curve $C$ uniquely, and thus is a convenient way to describe curves in $\mathbb{R}^{N} /\langle\mathbf{1}\rangle$. By abuse of notation, we will write the multiplicity $m\left(\sigma_{i}\right)$ also as $m\left(v_{i}\right)$ or $m\left(\left[v_{i}\right]\right)$.

We will now introduce the degree of a tropical 1-cycle and show that the set $P(C)$ gives a convenient way to compute it in the case of curves.

Definition 2.8 (Degree of a tropical 1-cycle). The degree $\operatorname{deg}(C)$ of a tropical 1-cycle $C$ in $\mathbb{R}^{n+1} /\langle\mathbf{1}\rangle$ is defined to be the (multiplicity of the origin in the) intersection product $L_{n-1}^{n} \cdot C$ of $C$ with a general tropical hyperplane.

Lemma 2.9 (The degree in terms of $P(C)$ ). Let $C \subset \mathbb{R}^{n+1} /\langle\mathbf{1}\rangle$ be a tropical curve. Then $\sum_{v \in P(C)} v=\operatorname{deg}(C) \cdot \mathbf{1}$.

Proof. As $L_{n-1}^{n}$ is cut out by the function $\varphi(x)=\min \left(x_{0}-x_{0}, x_{1}-x_{0}, \ldots, x_{n}-x_{0}\right)$, the intersection product $L_{n-1}^{n}$. $C$ is easily computed with the formula of Construction 2.2 for $\tau=\{0\}$ : the first term vanishes due to the balancing condition, and thus every 1-dimensional cone $\sigma$ in $C$ with corresponding vector $\left(x_{0}, \ldots, x_{n}\right)$ in $P(C)$, i. e. such that $m(\sigma) u_{\sigma}=\left[x_{0}, \ldots, x_{n}\right]$ and $\min \left(x_{0}, \ldots, x_{n}\right)=0$, gives rise to a contribution of

$$
-m(\sigma) \varphi\left(u_{\sigma}\right)=-\min \left(x_{0}-x_{0}, x_{1}-x_{0}, \ldots, x_{n}-x_{0}\right)=x_{0}
$$

to $L_{n-1}^{n} \cdot C$. In other words, the first coordinates of all vectors in $P(C)$ sum up to $\operatorname{deg}(C)$. Of course, by symmetry this means that the sum of all vectors in $P(C)$ is $\operatorname{deg}(C) \cdot \mathbf{1}$.

Example 2.10. For the tropical curve $C$ in $L_{2}^{3}$ from the picture in the introduction we have

$$
P(C)=\{(0,3,1,0),(0,0,1,3),(2,0,1,0),(1,0,0,0)\} \quad \subset \mathbb{Z}^{4} .
$$

As these vectors sum up to $(3,3,3,3)$, we see by Lemma 2.9 that $C$ has degree 3 .

For our applications we will need intersection products of $L_{2}^{3}$ with a classical plane. For this, let $a_{0}, a_{1}, a_{2}, a_{3} \in \mathbb{Z}$ not all zero with $a_{0}+\cdots+a_{3}=0$. We set $f: \mathbb{R}^{4} /\langle\mathbf{1}\rangle \rightarrow$ $\mathbb{R},\left(x_{0}, \ldots, x_{3}\right) \mapsto a_{0} x_{0}+\cdots+a_{3} x_{3}$ and $\varphi: \mathbb{R}^{4} /\langle\mathbf{1}\rangle \rightarrow \mathbb{R}, x \mapsto \min (0, f(x))$. Then the rational function $\varphi$ cuts out a cycle $H$ whose support is just the classical plane given by the equation $f=0$. We want to compute the intersection cycle $L_{2}^{3} \cdot H$.

Lemma 2.11 (Intersection products of $L_{2}^{3}$ with classical planes). With the notations as above, let $d=\sum_{i: a_{i}>0} a_{i}$. Then the set $P(C)$ for $C=L_{2}^{3} \cdot H$ consists exactly of the following vectors: 
(a) $a_{i} e_{j}-a_{j} e_{i}$ for all $i, j \in\{0,1,2,3\}$ with $a_{i}>0$ and $a_{j}<0$;

(b) $d e_{i}$ for all $i \in\{0,1,2,3\}$ with $a_{i}=0$.

In particular, we have $\operatorname{deg}(C)=d$.

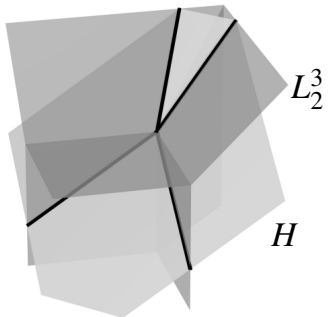

all $a_{i} \neq 0$, exactly two $a_{i}>0$

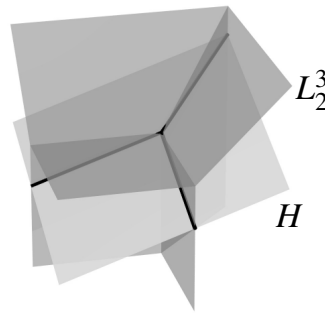

all $a_{i} \neq 0$, one or three $a_{i}>0$

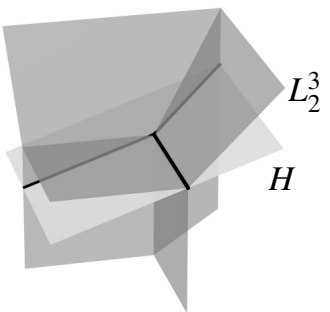

exactly one $a_{i}=0$

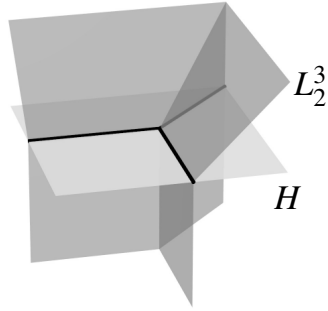

exactly two $a_{i}=0$

Proof. By Construction 2.2, the cones that can occur in the intersection product $L_{2}^{3} \cdot H$ are the 1-dimensional cones of $L_{2}^{3} \cap H$. As these are exactly the classes of the vectors listed in the lemma, it only remains to compute their multiplicities in $L_{2}^{3} \cdot H$.

Moreover, for all possibilities of the signs of $a_{0}, \ldots, a_{3}$, the vectors listed in the lemma sum up to $(d, d, d, d)$. Hence, they form a balanced cycle of degree $d$ by Lemma 2.9. As shown in the picture above, the number of these vectors can vary ( 3 or 4$)$, but in any case at most two of them are of type (b), i. e. point along a ray of $L_{2}^{3}$. Since $L_{2}^{3} \cdot H$ is a balanced cycle too and the balancing condition in the plane $H$ allows to reconstruct the multiplicities of up to two linearly independent cones, it thus suffices to check the multiplicities in the case (a).

In this case we can assume by symmetry that $i=0$ and $j=1$. Locally around the 1dimensional cone $\sigma$ spanned by $\left(-a_{1}, a_{0}, 0,0\right)$, the cycles $L_{2}^{3}$ and $H$ are then cut out by the rational functions $\min \left(0, x_{2}-x_{3}\right)$ and $\min \left(0, a_{0} x_{0}+\cdots+a_{3} x_{3}\right)$, respectively. In non-homogeneous coordinates with $x_{3}=0$ the corresponding functions are $\min \left(0, x_{2}\right)$ and $\min \left(0, a_{0} x_{0}+a_{1} x_{1}+a_{2} x_{2}\right)$. By [Rau08, Lemma 1.4] the multiplicity of $\sigma$ in the intersection product is therefore the index of the lattice $\left\{\left(x_{2}, a_{0} x_{0}+a_{1} x_{1}+a_{2} x_{2}\right): x_{0}, x_{1}, x_{2} \in \mathbb{Z}\right\}$ in $\mathbb{Z}^{2}$, i. e. the (positive) greatest common divisor of the $2 \times 2$ minors of the matrix

$$
\left(\begin{array}{ccc}
0 & 0 & 1 \\
a_{0} & a_{1} & a_{2}
\end{array}\right)
$$

which is just $\operatorname{gcd}\left(a_{0}, a_{1}\right)$. As desired, the vector in $P(C)$ corresponding to the cone $\sigma$ is thus $\operatorname{gcd}\left(a_{0}, a_{1}\right) \cdot \frac{1}{\operatorname{gcd}\left(a_{0}, a_{1}\right)}\left(-a_{1}, a_{0}, 0,0\right)=\left(-a_{1}, a_{0}, 0,0\right)$.

Construction 2.12 (Intersection products in $L_{2}^{3}$ ). Intersection products of cycles can not only be constructed in vector spaces, but also in matroid fans [FR10, Sha10]. In this paper we will only need the (degree of the) intersection product of two curves $C_{1}$ and $C_{2}$ in $L_{2}^{3}$; by [Sha10, Proposition 4.1] it is given by the explicit formula

$$
C_{1} \cdot C_{2}=\operatorname{deg}\left(C_{1}\right) \cdot \operatorname{deg}\left(C_{2}\right)-\sum_{0 \leq i<j \leq 3} \sum_{\substack{a e_{i}+b e_{j} \in P\left(C_{1}\right) \\ a, b>0}} \sum_{\substack{c e_{i}+d e_{j} \in P\left(C_{2}\right) \\ c, d>0}} \min (a d, b c) .
$$




\section{Projections of Matroid Fans}

In order to study the (relative) realizability of tropical curves in 2-dimensional matroid fans, our strategy is to use coordinate projections to map the situation to the plane, where we can then apply Newton polytope techniques. For example, there are four projections of the space $L_{2}^{3} \subset \mathbb{R}^{4} /\langle\mathbf{1}\rangle$ of Example 2.6 to the plane $\mathbb{R}^{3} /\langle\mathbf{1}\rangle$ which are described by forgetting one of the coordinates. But of course none of these projections is injective, and thus all of them lose some information on the curves in $L_{2}^{3}$. It is the main goal of this section to prove that all coordinate projections together suffice to reconstruct arbitrary tropical curves in the matroid fan (see Corollary 3.6).

Throughout this section, let $M$ be a loop-free matroid on a finite ground set $N$, and let $B(M) \subset \mathbb{R}^{N} /\langle\mathbf{1}\rangle$ be the corresponding matroid fan as in Construction 2.5, consisting of all cones $\sigma_{\mathscr{F}}$ for chains of flats $\mathscr{F}=\left(F_{1}, \ldots, F_{m}\right)$ in $M$. Recall that $\sigma_{\mathscr{F}}$ is generated by the vectors $\left[v_{F_{i}}\right]$, where $v_{F} \in \mathbb{R}^{N}$ for a flat $F$ has $i$-th coordinate 1 for $i \in F$, and 0 for $i \notin F$. For details on matroid theory we refer to [Ox192].

Construction 3.1 (Projections of matroid fans). For a non-empty subset $A \subset N$ we denote by $p^{A}: \mathbb{R}^{N} \rightarrow \mathbb{R}^{A}$ (and by abuse of notation also $p^{A}: \mathbb{R}^{N} /\langle\mathbf{1}\rangle \rightarrow \mathbb{R}^{A} /\langle\mathbf{1}\rangle$ ) the projection onto the coordinates of $A$. Our goal is to describe the projection $p^{A}(B(M))$.

For this we consider the so-called restricted matroid $\left.M\right|_{A}$ on $A$ whose independent sets are exactly those subsets of $A$ that are independent subsets of $N$ in $M$. It gives rise to a matroid fan $B\left(\left.M\right|_{A}\right) \subset \mathbb{R}^{A} /\langle\mathbf{1}\rangle$. We will now show that $p^{A}$ maps $B(M)$ to $B\left(\left.M\right|_{A}\right)$, and describe this map more precisely. An example of this is shown in the picture below, where $M$ is the uniform rank-3 matroid on $N=\{0,1,2,3\}$, so that $B(M)=L_{2}^{3}$, and $A=\{0,1,2\}$. Hence, $\left.M\right|_{A}$ is the uniform rank-3 matroid on $\{0,1,2\}$, the map $p^{A}$ just forgets the last coordinate, and can be viewed in the picture as the vertical projection onto $\mathbb{R}^{A} /\langle\mathbf{1}\rangle \cong \mathbb{R}^{2}$.

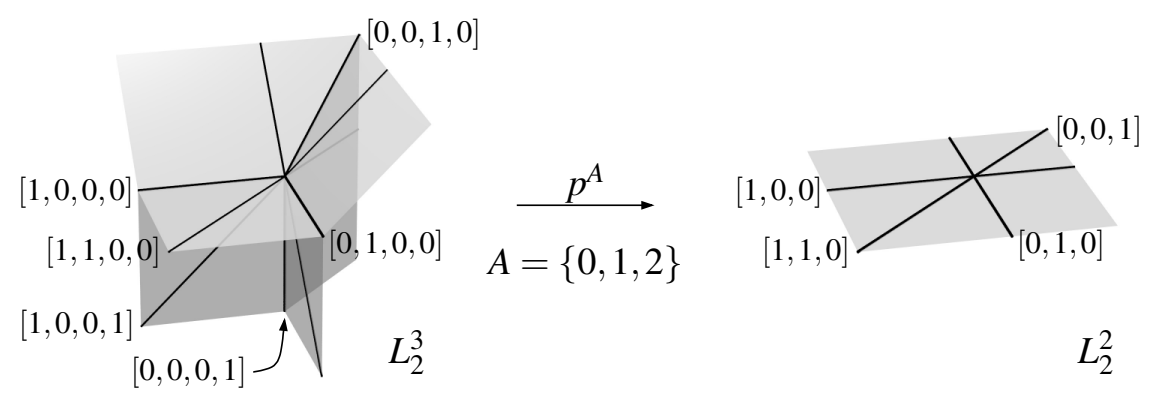

In order to describe $p^{A}$ we first note that, if $F \subset N$ is a flat of $M$, then $F \cap A \subset A$ is a flat of $\left.M\right|_{A}$ [Ox192, Proposition 3.3.1]. So if $\mathscr{F}=\left(F_{1}, \ldots, F_{m}\right)$ is a chain of flats in $M$ then $\left(F_{1} \cap A, \ldots, F_{m} \cap A\right)$ is a collection of ascending flats in $A-$ it might be however that some of these flats coincide or are equal to $\emptyset$ or $A$. We denote by $\mathscr{F} \cap A$ the chain of flats in $\left.M\right|_{A}$ obtained from the sequence $\left(F_{1} \cap A, \ldots, F_{m} \cap A\right)$ by deleting repeated entries and those that are equal to $\emptyset$ or $A$. In our example in the picture above, the chain of flats $\mathscr{F}=(\{0\},\{0,3\})$ in $M$ would e. g. give rise to the chain of flats $\mathscr{F} \cap A=(\{0\})$ in $\left.M\right|_{A}$.

With these notations we can now describe the projection $p^{A}$ as follows. 
Lemma 3.2 (Properties of projections of matroid fans). Let $A \subset N$ be a non-empty subset. With notations as in Constructions 2.5 and 3.1, we have for the corresponding projection $p^{A}: \mathbb{R}^{N} /\langle\mathbf{1}\rangle \rightarrow \mathbb{R}^{A} /\langle\mathbf{1}\rangle$ :

(a) $p^{A}\left(\left[v_{F}\right]\right)=\left[v_{F \cap A}\right]$ for every flat $F$ of $M$.

(b) Let $\mathscr{F}$ be a chain of flats in $M$. Then $p^{A}$ maps the corresponding cone $\sigma_{\mathscr{F}}$ of $B(M)$ surjectively to the cone $\sigma_{\mathscr{F} \cap A}$ of $B\left(\left.M\right|_{A}\right)$. The map $\left.p^{A}\right|_{\sigma_{\mathscr{F}}}$ is bijective if and only if the chains $\mathscr{F}$ and $\mathscr{F} \cap A$ have the same length.

(c) The maps $p^{A}$ and $\left.p^{A}\right|_{\sigma_{\mathscr{F}}}$ of (b) are also surjective and bijective, respectively, over $\mathbb{Z}$, i. e. they map $V_{\sigma_{\mathscr{F}}} \cap\left(\mathbb{Z}^{N} /\langle\mathbf{1}\rangle\right)$ surjectively and bijectively, respecively, to $V_{\sigma_{\mathscr{F} \cap A}} \cap$ $\left(\mathbb{Z}^{A} /\langle\mathbf{1}\rangle\right)$.

(d) $p^{A}$ maps $B(M)$ surjectively to $B\left(\left.M\right|_{A}\right)$.

(e) $p^{A}$ maps $B(M)$ surjectively to $\mathbb{R}^{A} /\langle\mathbf{1}\rangle$ if and only if $A$ is an independent set in $M$.

Proof. Statement (a) follows immediately from the definition of $v_{F}$, since $p^{A}$ just forgets the coordinates of $A$. So if $\mathscr{F}=\left(F_{1}, \ldots, F_{m}\right)$ is a chain of flats in $M$, the cone $\sigma_{\mathscr{F}}$ spanned by $\left[v_{F_{1}}\right], \ldots,\left[v_{F_{m}}\right]$ is mapped by $p^{A}$ surjectively to the cone spanned by $\left[v_{F_{1} \cap A}\right], \ldots,\left[v_{F_{m} \cap A}\right]$, which by definition is equal to $\sigma_{\mathscr{F} \cap A}$. As a linear map of cones this map is bijective if and only if $\sigma_{\mathscr{F}}$ and $\sigma_{\mathscr{F} \cap A}$ have the same dimension, i. e. if $\mathscr{F}$ and $\mathscr{F} \cap A$ have the same length. This shows (b). Statement (c) follows in the same way, noting that the lattices $V_{\sigma_{\mathscr{F}}} \cap\left(\mathbb{Z}^{N} /\langle\mathbf{1}\rangle\right)$ and $V_{\sigma_{\mathscr{F} \cap A}} \cap\left(\mathbb{Z}^{A} /\langle\mathbf{1}\rangle\right)$ are spanned by the classes of $v_{F_{1}}, \ldots, v_{F_{m}}$ and $v_{F_{1} \cap A}, \ldots, v_{F_{m} \cap A}$, respectively.

To show the last two statements, note that for every chain of flats $\mathscr{F}^{\prime}=\left(F_{1}^{\prime}, \ldots, F_{m}^{\prime}\right)$ in $\left.M\right|_{A}$ we get a chain of flats $\mathscr{F}=\left(\operatorname{cl}\left(F_{1}^{\prime}\right), \ldots, \mathrm{cl}\left(F_{m}^{\prime}\right)\right)$ in $M$ with $\mathscr{F} \cap A=\mathscr{F}^{\prime}$ by applying the closure operator cl of $M[\mathrm{Ox} 192,3.1 .16]$. Thus $p^{A}\left(\sigma_{\mathscr{F}}\right)=\sigma_{\mathscr{F}}$, and hence, the image of $p^{A}$ is all of $B\left(\left.M\right|_{A}\right)$, as claimed in (d). As $\mathbb{R}^{A} /\langle\mathbf{1}\rangle$ is irreducible [GKM09, chapter 2], this image $B\left(\left.M\right|_{A}\right)$ is equal to $\mathbb{R}^{A} /\langle\mathbf{1}\rangle$ if and only if its dimension is equal to $|A|-1$. This is the case if and only if the matroid $\left.M\right|_{A}$ has rank $|A|$, which in turn is equivalent to saying that $\left.M\right|_{A}$ is the uniform matroid on $A$, i. e. that $A$ is an independent set in $M$. This proves (e).

Definition 3.3 (Rank of a chain of flats). Let $r: \mathscr{P}(N) \rightarrow \mathbb{N}$ be the rank function of the matroid $M$, cf. [Ox192, Section 1.3]. For a chain of flats $\mathscr{F}=\left(F_{1}, \ldots, F_{m}\right)$ of $M$, with $\emptyset \subsetneq F_{1} \subsetneq \cdots \subsetneq F_{m} \subsetneq N$ as above, we define the rank of $\mathscr{F}$ to be

$$
r(\mathscr{F}):=r\left(F_{1}\right)+\cdots+r\left(F_{m}\right) .
$$

We will also call this the rank of the corresponding cone $\sigma_{\mathscr{F}}$ of $B(M)$.

Lemma 3.4. For each chain of flats $\mathscr{F}$ of $M$ there is a basis $A \subset N$ of $M$ such that:

(a) the projection $p^{A}$ is injective on the cone $\sigma_{\mathscr{F}}$ of $B(M)$;

(b) for every other chain of flats $\mathscr{F}^{\prime} \neq \mathscr{F}$ of $M$ with $p^{A}\left(\sigma_{\mathscr{F}}\right)=p^{A}\left(\sigma_{\mathscr{F}}\right)$ we have $r\left(\mathscr{F}^{\prime}\right)>r(\mathscr{F})$. 
Proof. Extend $\mathscr{F}=\left(F_{1}, \ldots, F_{m}\right)$ to a maximal chain of flats $\left(G_{1}, \ldots, G_{k}\right)$ of length $k:=$ $r(N)-1$ (with $G_{0}:=\emptyset \subsetneq G_{1} \subsetneq \cdots \subsetneq G_{k} \subsetneq N=: G_{k+1}$ ). Choosing an element of each $G_{i} \backslash G_{i-1}$ for $i=1, \ldots, k+1$, we then obtain a basis $A$ of $M$ with $r\left(G_{i} \cap A\right)=r\left(G_{i}\right)$ for all $i=1, \ldots, k$, and thus $r\left(F_{i}\right)=r\left(F_{i} \cap A\right)$ for all $i=1, \ldots, m$.

Hence, from $1 \leq r\left(F_{1}\right)<\cdots<r\left(F_{m}\right) \leq k$ it follows that $1 \leq r\left(F_{1} \cap A\right)<\cdots<r\left(F_{m} \cap A\right) \leq k$. So the sets $F_{1} \cap A, \ldots, F_{m} \cap A$ are all distinct and not equal to $\emptyset$ or $A$, and thus $\mathscr{F} \cap A=$ $\left(F_{1} \cap A, \ldots, F_{m} \cap A\right)$ and $r(\mathscr{F} \cap A)=r(\mathscr{F})$. In particular, since $\mathscr{F}$ and $\mathscr{F} \cap A$ have the same length it follows from Lemma 3.2 (b) that $p^{A}$ is injective on $\sigma_{\mathscr{F}}$. This shows part (a) of the lemma.

Now let $\mathscr{F}^{\prime}=\left(F_{1}^{\prime}, \ldots, F_{q}^{\prime}\right)$ be another chain of flats with the same image under $p^{A}$, i. e. by Lemma 3.2 (b) such that $\mathscr{F}^{\prime} \cap A=\mathscr{F} \cap A=\left(F_{1} \cap A, \ldots, F_{m} \cap A\right)$. Then for each $i=1, \ldots, m$ there must be an index $j_{i} \in\{1, \ldots, q\}$ with $F_{j_{i}}^{\prime} \cap A=F_{i} \cap A$, and thus

$$
r\left(\mathscr{F}^{\prime}\right) \geq \sum_{i=1}^{m} r\left(F_{j_{i}}^{\prime}\right) \geq \sum_{i=1}^{m} r\left(F_{j_{i}}^{\prime} \cap A\right)=\sum_{i=1}^{m} r\left(F_{i} \cap A\right)=r(\mathscr{F} \cap A)=r(\mathscr{F}) .
$$

In the case of equality $r\left(\mathscr{F}^{\prime}\right)=r(\mathscr{F})$ we must have $q=m$ (i. e. $j_{i}=i$ for all $i=1, \ldots, m$ ) and $r\left(F_{i}^{\prime}\right)=r\left(F_{i}^{\prime} \cap A\right)$ for all $i$. But then $F_{i}^{\prime}$ and $F_{i}$ are two flats of $M$ containing the set $F_{i}^{\prime} \cap A=F_{i} \cap A$, where

$$
r\left(F_{i}^{\prime}\right)=r\left(F_{i}^{\prime} \cap A\right)=r\left(F_{i} \cap A\right)=r\left(F_{i}\right) .
$$

This requires both $F_{i}^{\prime}$ and $F_{i}$ to be the closure of $F_{i}^{\prime} \cap A=F_{i} \cap A$ for all $i$. In particular, we then have $\mathscr{F}^{\prime}=\mathscr{F}$, completing the proof of (b).

Example 3.5. Let $M$ be the uniform rank-3 matroid on $N=\{0,1,2,3\}$, so $B(M)=L_{2}^{3}$ as in Example 2.6. Then $B(M)$ has cones of ranks 1, 2, and 3, corresponding to the following chains of flats:

(a) four 1-dimensional cones of rank 1 spanned by a unit vector, corresponding to the chains $\mathscr{F}=(\{i\})$ for $0 \leq i \leq 3$;

(b) six 1-dimensional cones of rank 2 spanned by a vector with two entries 1 and two entries 0 , corresponding to the chains $\mathscr{F}=(\{i, j\})$ for $0 \leq i<j \leq 3$;

(c) twelve 2 -dimensional cones of rank 3 , corresponding to the chains $\mathscr{F}=(\{i\},\{i, j\})$ for $0 \leq i, j \leq 3$ with $i \neq j$.

Let us apply (the proof of) Lemma 3.4 to the first type of chain, say to $\mathscr{F}=(\{0\})$ of rank 1 with corresponding cone $\sigma_{\mathscr{F}}$ spanned by $\left[v_{\{0\}}\right]=[1,0,0,0]$. We extend $\mathscr{F}$ to a maximal chain of flats, e. g. to $\emptyset \subsetneq\{0\} \subsetneq\{0,1\} \subsetneq N$, and derive from this the basis $A=\{0,1,2\}$ of $M$. Projecting $B(M)$ with $p^{A}$ (as in the picture in Construction 3.1) we see indeed that $\sigma_{\mathscr{F}}$ is mapped injectively to its image cone spanned by $[1,0,0]$, and that there are three more cones with the same image - namely the ones corresponding to the chains $(\{0,3\}),(\{0\},\{0,3\})$, and $(\{3\},\{0,3\})$ - and they all have bigger rank.

With this result we can now reconstruct arbitrary 1-cycles in matroid fans from their projections, by reconstructing their rays by descending induction on the rank of the cone in which they lie. 
Corollary 3.6 (Reconstruction of 1-cycles in matroid fans with projections). Let $C, C^{\prime} \in$ $Z_{1}^{\text {trop }}(B(M))$ be two 1-cycles contained in the Bergman fan $B(M)$.

If $p_{*}^{A}(C)=p_{*}^{A}\left(C^{\prime}\right)$ for all bases $A \subset N$ of $M$, then $C=C^{\prime}$.

Proof. Let $u \in \mathbb{Z}^{N} /\langle\mathbf{1}\rangle$ be a primitive vector contained in the support of $B(M)$, let $\sigma$ be the unique cone of $B(M)$ containing $u$ in its relative interior, and let $\mathscr{F}$ be the corresponding chain of flats of $M$. Moreover, let $\lambda$ and $\lambda^{\prime}$ be the multiplicities of $u$ in $C$ resp. $C^{\prime}$. We have to prove that $\lambda=\lambda^{\prime}$.

We will show this by descending induction on the rank $r(\mathscr{F})$ as in Definition 3.3. The start of the induction is trivial by Lemma 3.4, since the possible values of $r(\mathscr{F})$ for a given matroid fan are bounded. For the induction step choose a basis $A \subset N$ of $M$ for $\mathscr{F}$ as in Lemma 3.4, and let $w \in \mathbb{Z}^{A} /\langle\mathbf{1}\rangle$ be the primitive vector pointing in the direction of $p^{A}(u)$. Let $u_{1}, \ldots, u_{m} \in \mathbb{Z}^{N} /\langle\mathbf{1}\rangle$ be the primitive vectors occurring in $C$ or $C^{\prime}$ except $u$ that are mapped by $p^{A}$ to a positive multiple of $w$, and let $\lambda_{1}, \ldots, \lambda_{m}$ and $\lambda_{1}^{\prime}, \ldots, \lambda_{m}^{\prime}$ be their multiplicities in $C$ resp. $C^{\prime}$. Then the multiplicity of $w$ in $p_{*}^{A}(C)=p_{*}^{A}\left(C^{\prime}\right)$ is

$$
\lambda\left[\mathbb{Z} w: \mathbb{Z} p^{A}(u)\right]+\sum_{i=1}^{m} \lambda_{i}\left[\mathbb{Z} w: \mathbb{Z} p^{A}\left(u_{i}\right)\right]=\lambda^{\prime}\left[\mathbb{Z} w: \mathbb{Z} p^{A}(u)\right]+\sum_{i=1}^{m} \lambda_{i}^{\prime}\left[\mathbb{Z} w: \mathbb{Z} p^{A}\left(u_{i}\right)\right]
$$

by the definition of the push-forward of tropical cycles in Construction 2.3. Now Lemma 3.4 tells us that the vectors $u_{1}, \ldots, u_{m}$ must lie in cones of rank bigger than $r(\mathscr{F})$ (there can be no such vectors in $\mathscr{F}$ by part (a) of the lemma, and none in other cones of the same or smaller rank than $r(\mathscr{F})$ by (b)), and thus $\lambda_{i}=\lambda_{i}^{\prime}$ for all $i=1, \ldots, m$ by the induction assumption. Hence, we conclude by the above equation that $\lambda=\lambda^{\prime}$.

Remark 3.7. Note that by Lemma 3.2 (e) the required coordinate projections to reconstruct 1-cycles in $B(M)$ are precisely those that map $B(M)$ surjectively to a real vector space $\mathbb{R}^{A} /\langle\mathbf{1}\rangle$ of dimension $r(N)-1=\operatorname{dim} B(M)$. For example, in the case of $L_{2}^{3}$ of Example 2.6, corresponding to the uniform rank-3 matroid on $N=\{0,1,2,3\}$, we need the four coordinate projections $p^{A}: \mathbb{R}^{N} /\langle\mathbf{1}\rangle \rightarrow \mathbb{R}^{A} /\langle\mathbf{1}\rangle \cong \mathbb{R}^{2}$ for all subsets $A \subset N$ with $|A|=3$.

Remark 3.8 (Reconstruction in the non-constant coefficient case). In this paper we only consider the realizability by algebraic curves over a field with a trivial valuation. For curves over a non-Archimedean valued field, the so-called "non-constant coefficient case", one has to replace the 1-dimensional fan cycles of Construction 2.2 by 1-dimensional balanced polyhedral complexes modulo refinements as e. g. in [Rau09, Section 1.1]. For these more general cycles there is a push-forward along a tropical morphism $f$ as well: one first has to choose a subdivision of the given cycle which is fine enough for the images of its cells under $f$ to form a polyhedral complex. Then one assigns to each such 1-dimensional image cell $\tau^{\prime}$ the multiplicity $\sum_{\tau: f(\tau)=\tau^{\prime}} \lambda_{\tau}\left[\mathbb{Z} w: \mathbb{Z} f\left(u_{\tau}\right)\right]$, where $\lambda_{\tau}$ is the multiplicity of $\tau$, and $u_{\tau}$ and $w$ are primitive integer vectors in the directions of $\tau$ and $\tau^{\prime}$, respectively [Rau09, Section 1.3.2]. Comparing these multiplicities for two given cycles with the same arguments as in the proof above then shows that the statement of Corollary 3.6 also holds in this nonconstant coefficient setting. 
To conclude this section, we will prove that the push-forwards occurring in Construction 3.6 can easily be computed in terms of the sets $P(C)$ of Notation 2.7: To obtain $P\left(p_{*}^{A} C\right)$ one just has to delete the coordinates not corresponding to $A$ of the elements in $P(C)$ and then add up all vectors that are positive multiples of each other.

Lemma 3.9 (Projections of curves). Let $C$ be a tropical curve in $B(M)$, and let $A$ be a basis of $M$. Then the set $P\left(p_{*}^{A} C\right)$ consists exactly of the non-zero vectors of the form

$$
\sum_{v \in P(C):\left[p^{A}(v)\right] \in \sigma} p^{A}(v) \quad \in \mathbb{Z}^{A}
$$

for all 1-dimensional cones $\sigma$ in $\mathbb{R}^{A} /\langle\mathbf{1}\rangle$. In particular, we have $\operatorname{deg}\left(p_{*}^{A} C\right)=\operatorname{deg}(C)$.

Proof. We will show first that the vectors stated in the proposition satisfy the normalization requirement of Notation 2.7, i.e. that their minimal coordinate is 0 . Let $\sigma$ be a 1 dimensional cone in $\mathbb{R}^{A} /\langle\mathbf{1}\rangle$ and $v \in P(C)$ with $\left[p^{A}(v)\right] \in \sigma$. Moreover, let $\mathscr{F}$ and $\mathscr{F}^{\prime}$ be the unique chains of flats of $M$ and $\left.M\right|_{A}$, respectively, such that $[v]$ lies in the relative interior of the cone $\sigma_{\mathscr{F}}$ of $B(M)$ and $\sigma \backslash\{0\}$ lies in the relative interior of the cone $\sigma_{\mathscr{F}}$ of $B\left(\left.M\right|_{A}\right)$. By Lemma 3.2 (b) we then have $p^{A}\left(\sigma_{\mathscr{F}}\right)=\sigma_{\mathscr{F}}$, and thus $\mathscr{F}^{\prime}=\mathscr{F} \cap A$. Now choose $i \in A$ not contained in any flat in $\mathscr{F}^{\prime}$. Then $i$ cannot be contained in any flat $F$ of $\mathscr{F}$ either, because otherwise $\mathscr{F}^{\prime}=\mathscr{F} \cap A$ implies $A \subset F$ and hence $r(F) \geq r(A)=r(N)$, yielding the contradiction $F=N$. By Construction 2.5 this means that the $i$-th coordinate is minimal, and thus 0 , in $v$. As $i$ does not depend on $v$ but only on $\sigma$, it follows that the minimal coordinate is 0 in all the vectors stated in the lemma.

Next, let us check that the multiplicity of each cone $\sigma$ as above in $p_{*}^{A} C$ is correct. As the minimal coordinate of each $v \in P(C)$ with $\left[p^{A}(v)\right] \in \sigma$ is 0 , by Construction 2.3 the cone $\sigma$ receives a multiplicity of

$m_{C}(v) \cdot\left[\mathbb{R} p^{A}(u) \cap\left(\mathbb{Z}^{A} /\langle\mathbf{1}\rangle\right): \mathbb{Z} p^{A}(u)\right]=\operatorname{gcd}\left\{v_{i}: i \in N\right\} \cdot \frac{\operatorname{gcd}\left\{v_{i}: i \in A\right\}}{\operatorname{gcd}\left\{v_{i}: i \in N\right\}}=\operatorname{gcd}\left\{v_{i}: i \in A\right\}$

from $v$, where $u$ denotes the primitive integral vector in $\mathbb{Z}^{N} /\langle\mathbf{1}\rangle$ in the direction of $[v]$. But as the minimal coordinate of $p^{A}(v)$ is 0 as well, this is exactly the integer length of $\left[p^{A}(v)\right]$ in $\mathbb{Z}^{A} /\langle\mathbf{1}\rangle$. So the multiplicities of the rays are indeed correct.

Finally, the statement about the degree now follows immediately from Lemma 2.9.

\section{RELATIVE REALIZABILITY}

4.1. Tropicalization. As explained in the introduction, we are concerned with a particular tropical inverse problem, i. e. a question of which tropical cycles are realizable as tropicalizations of algebraic varieties satisfying certain given conditions. To describe our relative realization problem, we will use the following setup.

Notation 4.1. Throughout this section, let $K$ be an algebraically closed field, and fix $n \in \mathbb{N}$. We denote by $R=K\left[x_{0}, \ldots, x_{n}\right]$ the polynomial ring, and by $S=K\left[x_{0}^{ \pm 1}, \ldots, x_{n}^{ \pm 1}\right]$ the Laurent polynomial ring in $n+1$ variables over $K$. Consider the standard grading on $R$ and $S$, and let $S_{0}=K\left[\frac{x_{i}}{x_{j}}: 0 \leq i, j \leq n\right]$ be the $K$-algebra of elements of degree 0 in $S$. Let $X=\operatorname{Spec}\left(S_{0}\right)$ 
be the $n$-dimensional torus over $K$. Algebraic varieties and curves are always assumed to be irreducible.

Construction 4.2 (Tropicalization of varieties). Let $X$ be the torus as in Notation 4.1. We define a tropicalization of subvarieties of $X$ to $\mathbb{R}^{n+1} /\langle\mathbf{1}\rangle$ as follows. Let $f=\sum_{v} a_{v} x^{v} \in S_{0}$ with $a_{v} \in K$ (for $v \in \mathbb{Z}^{n+1}$ such that $\sum_{i} v_{i}=0$ ). For $\omega \in \mathbb{R}^{n+1}$ let $c_{\omega}=\min _{v: a_{v} \neq 0} \omega \cdot v$, where we use the standard scalar product on $\mathbb{R}^{n+1}$. Then we $\operatorname{set}_{\omega}(f)=\sum_{v: \omega \cdot v=c_{\omega}} a_{v} x^{v}$ to be the initial polynomial with respect to $\omega$, and for an ideal $I \subset S_{0}$ we set $\operatorname{in}_{\omega}(I)=\left(\operatorname{in}_{\omega}(f): f \in I\right)$.

For a subvariety $Y \subset X$ given by a prime ideal $P \subset S_{0}$ we consider the set $\operatorname{trop}(Y)=$ $\left\{\omega \in \mathbb{R}^{n+1}: \operatorname{in}_{\omega}(P) \neq S_{0}\right\}$. This is the underlying set of a polyhedral fan in $\mathbb{R}^{n+1}$ induced by the Gröbner fan of $P S \cap R$. For every maximal cone $\sigma$ in this fan we define a multiplicity

$$
m(\sigma)=\sum_{\operatorname{in}_{\omega}(P) \subset Q} \ell\left(\left(S_{0} / \text { in }_{\omega}(P)\right)_{Q}\right),
$$

where $\omega$ is any element of the relative interior of $\sigma$, the sum is taken over all minimal primes $Q$ of $\operatorname{in}_{\omega}(P)$, and $\ell(M)$ denotes the length of the $S_{0}$-module $M$. By [Spe05] we know that $\operatorname{trop}(Y)$ is a balanced polyhedral fan with these multiplicities, and thus a tropical variety in the sense of Notation 2.1.

As $\langle\mathbf{1}\rangle$ is contained in the lineality space of $\operatorname{trop}(Y)$ it is in fact more natural to consider $\operatorname{trop}(Y)$ in $\mathbb{R}^{n+1} /\langle\mathbf{1}\rangle$. Hence, from now on, by the tropicalization $\operatorname{trop}(Y)$ of $Y$ we will always mean this balanced polyhedral fan in $\mathbb{R}^{n+1} /\langle\mathbf{1}\rangle$. We have $\operatorname{dim} Y=\operatorname{dim} \operatorname{trop}(Y)$ after this identification by [Spe05, Theorem 2.1.2] together with the first main result of [BG86].

Example 4.3 (Linear varieties). Let $L$ be a linear ideal in $S$, i. e. an ideal that can be generated by $n-k$ independent linear forms $l_{1}, \ldots, l_{n-k}$ in $x_{0}, \ldots, x_{n}$, where $k+1=\operatorname{dim}(S / L)$. With $L_{0}=L \cap S_{0}$ we then have $\operatorname{dim}\left(S_{0} / L_{0}\right)=k$. We will call $E=\operatorname{Spec}\left(S_{0} / L_{0}\right)$ a $k$-dimensional linear variety in the torus $X=\operatorname{Spec}\left(S_{0}\right)$.

The tropicalization of $E$ is easy to describe: let $Q$ be a $(k+1) \times(n+1)$ matrix whose rows span the zero set of $\left(l_{1}, \ldots, l_{n-k}\right)$, and denote by $M(L)$ the matroid on the columns of $Q$ in the sense of [Ox192, Proposition 1.1.1]. Then $\operatorname{trop}(E)$ is exactly the associated matroid fan $B(M(L))$ as in Construction 2.5, see [AK06, Theorem 1]. For example, if $L$ and thus also $Q$ is general, we obtain for $M(L)$ the uniform matroid of rank $k+1$ on $n+1$ elements, and consequently $\operatorname{trop}(E)=B(M(L))=L_{k}^{n}$ as in Example 2.6.

In the case of curves, let us extend the tropicalization map from varieties to cycles.

Definition 4.4 (Tropicalization of 1-cycles). Let $E$ be a subvariety of the torus $X$.

(a) We denote by $Z_{1}(E)$ the group of 1-cycles in $E$ in the sense of [Ful98], i. e. the free abelian group generated by all curves in $E$. Moreover, let $Z_{1}^{+}(E) \subset Z_{1}(E)$ be the subset of effective cycles. By abuse of notation, for a curve $Y \subset E$ we will also write $Y \in Z_{1}^{+}(E)$ for the 1-cycle whose multiplicity is 1 on $Y$ and 0 on all other curves.

(b) For $Y \in Z_{1}(E)$ we define the degree $\operatorname{deg}(Y) \in \mathbb{Z}$ of $Y$ to be the intersection product of $Y$ with a general hyperplane in $X$. The subsets of $Z_{1}(E)$ and $Z_{1}^{+}(E)$ of cycles of degree $d$ will be denoted $\left(Z_{1}(E)\right)_{d}$ resp. $\left(Z_{1}^{+}(E)\right)_{d}$. 
(c) We extend the tropicalization of Construction 4.2 by linearity to a group homomorphism

$$
\text { Trop : } Z_{1}(E) \longrightarrow Z_{1}^{\text {trop }}(\operatorname{trop}(E)) \text {. }
$$

Lemma 4.5 (Tropicalization preserves the degree). For any subvariety $E \subset X$ and cycle $Y \in Z_{1}(E)$ we have $\operatorname{deg}(\operatorname{Trop}(Y))=\operatorname{deg}(Y)$, with the tropical degree as in Definition 2.8.

Proof. By linearity it suffices to prove the statement for a curve $Y \subset E$. Let $G$ be a general hyperplane in $X$, so that $\operatorname{trop}(G)=L_{n-1}^{n}$ by Example 4.3. Moreover, let $\Delta$ be a complete unimodular fan in $\mathbb{R}^{n+1} /\langle\mathbf{1}\rangle$ containing $\operatorname{trop}(Y)$ and trop $(G)$ as subfans. Then the corresponding toric variety $X(\Delta)$ is complete and smooth. Hence, by [FS97, Theorem 3.1] there is a natural ring homomorphism $\phi: A_{*}(X(\Delta)) \rightarrow Z_{*}^{\text {trop }}\left(\mathbb{R}^{n+1} /\langle\mathbf{1}\rangle\right)$, where $A_{*}(X(\Delta))$ denotes the Chow homology of $X(\Delta)$, and the ring structures are given by the algebraic resp. tropical intersection product. By the fundamental theorem of tropical geometry [Spe05] together with [ST08, Corollary 3.15] we know that $\phi([\bar{W}])=\operatorname{trop}(W)$ for every $W \subset X$ such that $\operatorname{trop}(W)$ is a subfan of $\Delta$. In particular,

$$
\phi([\bar{G}] \cdot[\bar{Y}])=\phi([\bar{G}]) \cdot \phi([\bar{Y}])=L_{n-1}^{n} \cdot \operatorname{trop}(Y)=\operatorname{deg}(\operatorname{trop}(Y)),
$$

where we identify $Z_{0}^{\text {trop }}\left(\mathbb{R}^{n+1} /\langle\mathbf{1}\rangle\right)$ with $\mathbb{Z}$. But $\bar{G}$ and $\bar{Y}$ only intersect in the torus $X$ of $X(\Delta)$, since $G$ is general. So we have $\operatorname{deg}([\bar{G}] \cdot[\bar{Y}])=\operatorname{deg}(Y)$, and the result follows.

In this paper we will always consider curves or 1-cycles in a fixed 2-dimensional linear variety in $X$. So let us fix the following notation.

Notation 4.6 (Planes). In the following, let $L=\left(l_{1}, \ldots, l_{n-2}\right)$ always be a linear ideal in $S$ with $\operatorname{dim}(S / L)=3$ as in Example 4.3, and let $L_{0}=L \cap S_{0}$. Then $E:=\operatorname{Spec}\left(S_{0} / L_{0}\right)$ is a plane in the torus $X$, and $\operatorname{trop}(E)=B(M(L))$ will be called a tropical plane. By abuse of notation, the ideal $L \cap R$ in $R$ will also be denoted by $L$.

Construction $4.7\left(\left(Z_{1}^{+}(E)\right)_{d}\right.$ as an algebraic variety). We now want to give the sets $\left(Z_{1}^{+}(E)\right)_{d}$ of effective 1-cycles of degree $d$ in the plane $E$ the structure of an algebraic variety.

By [Har77, Proposition 6.11], every effective 1-cycle $Y$ in $E$ is the divisor in $E$ of a regular function on $E$, i. e. of an element of $S_{0} / L_{0}$, unique up to units. Hence, $Z_{1}^{+}(E)$ is in natural bijection with the set of principal ideals in $S_{0} / L_{0}$. As $S_{0} / L_{0}$ is just the degree-0 part of $S / L$, this set corresponds by extension to the set of principal homogeneous ideals in $S / L$, which in turn by localization corresponds to the set of principal ideals in $R / L$ generated by a homogeneous polynomial without monomial factors [Eis95, Proposition 2.2]. Let $f^{Y} \in R / L$ be a homogeneous polynomial such that the ideal $\left(f^{Y}\right) \subset R / L$ corresponds to $Y \in Z_{1}^{+}(E)$ in this way. Note that the choice of $f^{Y}$ is unique up to multiplication with an element of $K^{*}$.

Geometrically, the plane $E$ is a dense open subset of the projective $\operatorname{space} \operatorname{Proj}(R / L) \cong \mathbb{P}^{2}$. So by taking the closure, a cycle $Y \in Z_{1}^{+}(E)$ determines a cycle $\bar{Y} \in Z_{1}^{+}(\operatorname{Proj}(R / L))$ without components in coordinate hyperplanes, which is just the divisor of $f^{Y}$. In particular, by Bézout's theorem we see that $\operatorname{deg} f^{Y}=\operatorname{deg} Y$. So we get an injective map

$$
\left(Z_{1}^{+}(E)\right)_{d} \longleftrightarrow \mathbb{P}\left((R / L)_{d}\right), Y \mapsto\left[f^{Y}\right]
$$


to a projective space of dimension $\frac{d(d+3)}{2}$, where $(R / L)_{d}$ denotes the degree- $d$ part of $R / L$. As explained above, its image is the complement of the space of polynomials without monomial factors, and thus a dense open subset. As $\mathbb{P}\left((R / L)_{d}\right)$ is irreducible, this gives $\left(Z_{1}^{+}(E)\right)_{d}$ the structure of an open subvariety of $\mathbb{P}\left((R / L)_{d}\right)$. In the following we will always consider $\left(Z_{1}^{+}(E)\right)_{d}$ as an algebraic variety in this way.

It is now the goal of this paper to study which tropical curves in $\operatorname{trop}(E)$ can be realized as tropicalizations of effective 1-cycles in $E$, and to describe the space of such cycles.

Definition 4.8 (Relative realizability and realization space). Let $E$ be a plane in the torus $X$ defined by a linear ideal $L$ as in Notation 4.6. Moreover, let $C \in Z_{1}^{\text {trop }}(\operatorname{trop}(E))$ be a tropical curve of degree $d$ as in Notation 2.1 and Definition 2.8.

(a) We say that $C$ is (relatively) realizable in $E$ (or in $L$ ) if there exists an effective cycle $Y \in Z_{1}^{+}(E)$ with $\operatorname{Trop}(Y)=C$ (note that we must have $\operatorname{deg}(Y)=d$ in this case by Lemma 4.5).

(b) The subset $\operatorname{Real}(C) \subset\left(Z_{1}^{+}(E)\right)_{d}$ of all effective cycles $Y$ such that $\operatorname{Trop}(Y)=C$ is called the (relative) realization space of $C$. We will see in Algorithm 4.15 that $\operatorname{Real}(C)$ is the complement of a union of hyperplanes in a linear space; its dimension will be called the realization dimension realdim $(C)$ of $C$.

4.2. Projecting to the plane. As realizability is completely understood in the plane case (see Lemma 4.14), one idea to deal with more complicated inverse problems is to reduce them to questions about this case. In this section we will give an equivalent description of our realization problem in terms of several dependent realization problems in the plane, which can then be attacked algorithmically.

To relate our problem to the plane case, we will use coordinate projections onto the plane which preserve enough information both on the algebraic and the tropical side, such that from all images of those projections we can reconstruct the original objects. By Corollary 3.6 these can be chosen to be all projections onto coordinates indexed by the bases of the matroid $M(L)$. On the algebraic side such a projection is simply a monomorphism and can geometrically be imagined as pushing the plane $E$ in space injectively to a coordinate plane. It can be described as follows.

Construction 4.9 (Algebraic projections to the plane). Let $A=\left\{j_{0}, j_{1}, j_{2}\right\} \subset\{0, \ldots, n\}$ be a basis of $M(L)$. Let $R^{A}=K\left[x_{j_{0}}, x_{j_{1}}, x_{j_{2}}\right]$ and $S_{0}^{A}=K\left[\frac{x_{i}}{x_{k}}: i, k=j_{0}, j_{1}, j_{2}\right]$, and consider the $K$-algebra monomorphism $S_{0}^{A} \rightarrow S_{0} / L_{0}$ mapping $\frac{x_{i}}{x_{k}}$ to $\frac{x_{i}}{x_{k}}+L_{0}$. This defines an injective morphism $\pi^{A}$ of affine varieties from $E=\operatorname{Spec}\left(S_{0} / L_{0}\right)$ to the 2-dimensional torus $E^{A}:=$ $\operatorname{Spec}\left(S_{0}^{A}\right)$, and thus an injective group homomorphism

$$
\pi_{*}^{A}: Z_{1}(E) \longleftrightarrow Z_{1}\left(\pi^{A}(E)\right) \longleftrightarrow Z_{1}\left(E^{A}\right)
$$

of the corresponding cycle groups. Note that $\pi_{*}^{A}$ preserves degrees and effective cycles, and thus maps $\left(Z_{1}^{+}(E)\right)_{d} \subset \mathbb{P}\left((R / L)_{d}\right)$ to $\left(Z_{1}^{+}\left(E^{A}\right)\right)_{d} \subset \mathbb{P}\left(R_{d}^{A}\right)$. To describe $\pi_{*}^{A}$ explicitly in 
terms of these ambient spaces, note that $A$ being a basis of $M(L)$ implies that the $K$-algebra homomorphism of polynomial rings

$$
R^{A} \rightarrow R / L, x_{i} \mapsto \overline{x_{i}}
$$

inducing the map $S_{0}^{A} \rightarrow S_{0} / L_{0}$ from above is an isomorphism. There is thus an inverse morphism

$$
R / L \rightarrow R^{A}, \overline{x_{i}} \mapsto a_{i, j_{0}} x_{j_{0}}+a_{i, j_{1}} x_{j_{1}}+a_{i, j_{2}} x_{j_{2}}
$$

with unique $a_{i, j} \in K$ such that $x_{i}-a_{i, j_{0}} x_{j_{0}}-a_{i, j_{1}} x_{j_{1}}-a_{i, j_{2}} x_{j_{2}} \in L$ for all $i$. It maps the class of a polynomial $f \in R$ to the polynomial $f_{A} \in R^{A}$ obtained from $f$ by replacing $x_{i}$ with $a_{i, j_{0}} x_{j_{0}}+a_{i, j_{1}} x_{j_{1}}+a_{i, j_{2}} x_{j_{2}}$ for all $i$, i. e. by eliminating all $x_{i}$ with $i \notin A$ modulo $L$. The map $\pi_{*}^{A}:\left(Z_{1}^{+}(E)\right)_{d} \rightarrow\left(Z_{1}^{+}\left(E^{A}\right)\right)_{d}$ is thus obtained by restriction from the isomorphism

$$
\mathbb{P}\left((R / L)_{d}\right) \rightarrow \mathbb{P}\left(R_{d}^{A}\right),[\bar{f}] \mapsto\left[f_{A}\right] .
$$

The main idea of our algorithm deciding the relative realizability problem relies on the fact that projection commutes with tropicalization. More precisely, we have the following commutative diagram described in [ST08, Theorem 1.1]:

Theorem 4.10 (Sturmfels-Tevelev). For every basis A of $M(L)$ the diagram

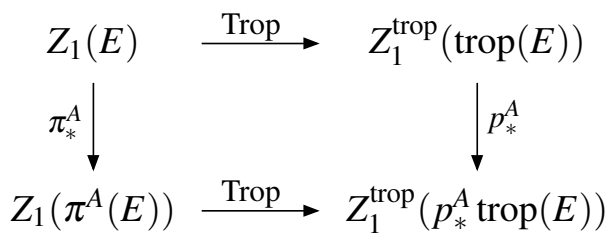

commutes, where $p^{A}$ is the projection as in Construction 3.1.

Note that all maps in this diagram preserve the degree of cycles by Lemma 3.9 and Lemma 4.5. Moreover, note that $p_{*}^{A}(\operatorname{trop}(E))$ is simply the plane $\mathbb{R}^{A} /\langle\mathbf{1}\rangle \cong \mathbb{R}^{2}$. We can thus reduce our relative realizability problem to a finite number of dependent realizability problems in the plane case, as stated in the following theorem.

Theorem 4.11 (Tropicalization by projections). Let $C \subset \operatorname{trop}(E)$ be a 1-dimensional tropical cycle, and let $Y \in Z_{1}(E)$. Then the following are equivalent:

(a) $\operatorname{Trop}(Y)=C$,

(b) $\operatorname{Trop}\left(\pi_{*}^{A}(Y)\right)=p_{*}^{A}(C)$ for every basis $A$ of $M(L)$.

Proof. By Theorem 4.10 it is clear that (a) $\Rightarrow($ b). We show (b) $\Rightarrow$ (a): For every basis $A$ of $M(L)$ we have $\operatorname{Trop}\left(\pi_{*}^{A}(Y)\right)=p_{*}^{A}(\operatorname{Trop}(Y))$ by Theorem 4.10. Hence, $p_{*}^{A}(\operatorname{Trop}(Y))=$ $p_{*}^{A}(C)$ for every basis $A$ for the two 1-cycles $\operatorname{Trop}(Y)$ and $C$, which are both contained in trop $(E)$. By Corollary 3.6 this implies $\operatorname{Trop}(Y)=C$. 
4.3. The plane case: Newton polytopes. To study the realization problem in the plane $\mathbb{R}^{3} /\langle\mathbf{1}\rangle$ we can use Newton polytopes. To describe this setup, let us consider the case $n=2$, i. e. $R=K\left[x_{0}, x_{1}, x_{2}\right], S_{0}=K\left[\left(\frac{x_{1}}{x_{0}}\right)^{ \pm 1},\left(\frac{x_{2}}{x_{0}}\right)^{ \pm 1}\right], E=X=\operatorname{Spec}\left(S_{0}\right)$ is the 2-dimensional torus, and thus $\operatorname{trop}(E)=\mathbb{R}^{3} /\langle\mathbf{1}\rangle$. Moreover, let $H=\left\{v \in \mathbb{R}^{3}: v_{0}+v_{1}+v_{2}=0\right\}$ be the vector space dual to $\mathbb{R}^{3} /\langle\mathbf{1}\rangle$, with dual lattice $\left\{v \in \mathbb{Z}^{3}: v_{0}+v_{1}+v_{2}=0\right\}$. For $d \in \mathbb{N}$ we set $H_{d}=\left\{v \in \mathbb{R}^{3}: v_{0}+v_{1}+v_{2}=d\right\}$ and $\Delta_{d}=\operatorname{conv}\{(d, 0,0),(0, d, 0),(0,0, d)\} \subset H_{d}$.

Construction 4.12 (Inner normal fans). Let $P$ be a lattice polytope in $H_{d}$ for some $d \in \mathbb{N}$ with $\operatorname{dim} P \geq 1$. We consider the inner normal fan $N(P) \subset \mathbb{R}^{3} /\langle\mathbf{1}\rangle$ of $P$ in the sense of [Zie02, Example 7.3], possibly refined such that the origin is a cone. For each 1-dimensional cone $\sigma$ in $N(P)$ we define the multiplicity $m(\sigma)$ to be the lattice length of the corresponding edge of $P$. Then $N(P)$ is a tropical curve in $\mathbb{R}^{3} /\langle\mathbf{1}\rangle$, and for a given $d$ the assignment $P \mapsto N(P)$ yields a one-to-one correspondence between lattice polytopes in $H_{d}$ of dimension at least 1 up to translation and plane tropical curves [Mik02, Corollary 2.5].

Definition 4.13 (Newton polytopes of polynomials, 1-cycles, and tropical curves).

(a) The Newton polytope of a homogeneous polynomial $f=\sum_{v} a_{v} x^{v} \in R$ of degree $d$ is defined to be $\operatorname{Newt}(f)=\operatorname{conv}\left\{v: a_{v} \neq 0\right\} \subset \Delta_{d} \subset H_{d}$.

(b) The Newton polytope $\operatorname{Newt}(Y)$ of an effective 1-cycle $Y \in Z_{1}^{+}(E)$ of degree $d$ is defined to be the Newton polytope of a polynomial $f \in R_{d}$ corresponding to $Y$ via the inclusion $\left(Z_{1}^{+}(E)\right)_{d} \subset \mathbb{P}\left(R_{d}\right)$ of Construction 4.7 .

(c) The Newton polytope $\operatorname{Newt}(C)$ of a tropical curve $C$ in $\mathbb{R}^{3} /\langle\mathbf{1}\rangle$ is the unique lattice polytope in $\mathbb{R}^{3}$ such that

- the inner normal fan of $\operatorname{Newt}(C)$ is $C$ (this fixes the polytope up to translation by Construction 4.12),

- $\operatorname{Newt}(C) \subset \Delta_{d}$ and meets all three sides of $\Delta_{d}$ for some $d$.

Lemma 4.14 (Tropicalization of plane 1-cycles). Let $Y \in Z_{1}^{+}(E)$ and $C \in Z_{1}^{\text {trop }}\left(\mathbb{R}^{3} /\langle\mathbf{1}\rangle\right)$. Then $\operatorname{Trop}(Y)=C$ if and only if $\operatorname{Newt}(Y)=\operatorname{Newt}(C)$.

In particular, every tropical curve in $\mathbb{R}^{3} /\langle\mathbf{1}\rangle$ is realizable, and the number $d$ in Definition 4.13 (c) is the degree of $C$.

Proof. By [EKL06, Corollary 2.1.2] the tropicalization of $Y$ is just the inner normal fan of $\operatorname{Newt}(Y)$. So if $\operatorname{Newt}(Y)=\operatorname{Newt}(C)$ then $\operatorname{Trop}(Y)$ equals the inner normal fan of $\operatorname{Newt}(C)$, which is $C$. Conversely, if $\operatorname{Trop}(Y)=C$, the inner normal fan of $\operatorname{Newt}(Y)$ is $C$. Moreover, this polytope is the Newton polytope of a homogeneous polynomial without monomial factors by Construction 4.7, so it is contained in $\Delta_{d}$ with $d=\operatorname{deg}(Y)$ and meets all three sides of $\Delta_{d}$. Hence, $\operatorname{Newt}(Y)=\operatorname{Newt}(C)$ by Definition 4.13 (c).

In particular, if $C$ is a tropical curve in $\mathbb{R}^{3} /\langle\mathbf{1}\rangle$ with $\operatorname{Newt}(C) \subset \Delta_{d}$ we can choose a homogeneous polynomial of degree $d$ with Newton polytope $\operatorname{Newt}(C)$. As this polynomial then does not have a monomial factor, it determines a cycle $Y \in\left(Z_{1}^{+}(E)\right)_{d}$ with $\operatorname{Newt}(Y)=\operatorname{Newt}(C)$, and thus with $\operatorname{Trop}(Y)=C$. So $C$ is realizable, and by Lemma 4.5 we have $d=\operatorname{deg}(Y)=\operatorname{deg}(C)$. 
Note that this result gives us explicit conditions on an effective 1-cycle realizing a tropical curve. This will play an important role in our algorithm.

4.4. Computing Realizability. Let us now collect our results to obtain an algorithm to detect realizability and compute the realization space and dimension of a curve $C$ in a tropical plane trop $(E)$.

Note that $C$ can only be realized by effective cycles of degree $d=\operatorname{deg}(C)$ by Lemma 4.5. So to deal with our problem algorithmically we first of all need to choose coordinates on the space $\left(Z_{1}^{+}(E)\right)_{d}$ of such cycles. This is easily achieved, since we have $\left(Z_{1}^{+}(E)\right)_{d} \subset \mathbb{P}\left((R / L)_{d}\right)$ as an open subset by Construction 4.7 , and $\mathbb{P}\left((R / L)_{d}\right) \cong \mathbb{P}\left(R_{d}^{B}\right)$ for a basis $B$ of the matroid $M(L)$ and $R=K\left[x_{i}: i \in B\right]$ by Construction 4.9. So we can choose homogeneous coordinates for the projective space $\mathbb{P}\left(R_{d}^{B}\right)$, i. e. the coefficients of a homogeneous polynomial of degree $d$ in three variables $x_{i}$ with $i \in B$, as homogeneous coordinates for $\left(Z_{1}^{+}(E)\right)_{d}$. The resulting algorithm to detect realizability and compute the realization space and dimension can be described as follows.

Algorithm 4.15 (Realizability of curves in a tropical plane). Consider a plane $E \subset X$ given by a linear ideal $L \subset S$ with $\operatorname{dim}(S / L)=3$, and let $C$ be a tropical curve in $\operatorname{trop}(E)$.

(a) Compute the degree of $C$ : by Lemma 2.9 this is just the natural number $d$ such that $\sum_{v \in P(C)} v=d \cdot \mathbf{1}$.

(b) Compute a basis $B=\left\{j_{0}, j_{1}, j_{2}\right\}$ of the matroid $M(L)$ associated to $L$.

(c) Let

$$
f=\sum_{v \in \mathbb{N}^{3},|v|=d} a_{v} x^{v} \in K\left[x_{j_{0}}, x_{j_{1}}, x_{j_{2}}\right],
$$

where the $a_{v}$ are parameters in $K$ that form the coordinates of the projective space $\mathbb{P}\left(R_{d}^{B}\right)$ containing our moduli space $\left(Z_{1}^{+}(E)\right)_{d}$ as explained above. More precisely, we can consider $f$ to be in the polynomial ring

$$
K\left[a_{v}: v \in \mathbb{N}^{3},|v|=d\right]\left[x_{j_{0}}, x_{j_{1}}, x_{j_{2}}\right] .
$$

(d) For every basis $A$ of $M(L)$ compute the polynomial $f_{A} \in K\left[x_{i}: i \in A\right]$ as in Construction 4.9 by eliminating all $x_{i}$ with $i \notin A$ from $f$ modulo $L$. Note that the coefficients of $f_{A}$ are linear forms in the $a_{v}$ determined by $L$.

(e) On the other hand, for every basis $A$ compute the tropical push-forward $C_{A}=p_{*}^{A}(C)$ by Lemma 3.9, and its Newton polytope $\operatorname{Newt}\left(C_{A}\right)$ as in Definition 4.13 (c). This can e. g. be done explicitly by Lemma 5.5 . Note that $\operatorname{deg}\left(C_{A}\right)=d$ for all $A$ by Lemma 3.9, and thus $\operatorname{Newt}\left(C_{A}\right) \subset \Delta_{d}$ by Lemma 4.14.

(f) Obtain conditions on the $a_{v}$ to ensure that $\operatorname{Newt}\left(f_{A}\right)=\operatorname{Newt}\left(C_{A}\right)$ for all bases $A$ : if $f_{A}=\sum_{v} b_{v} x^{v}$, this means that $b_{v}=0$ if $v \notin \operatorname{Newt}\left(C_{A}\right)$, whereas $b_{v} \neq 0$ if $v$ is a vertex of $\operatorname{Newt}\left(C_{A}\right)$. On the other coefficients of $f_{A}$ there are no conditions. This gives a set of linear equalities and non-equalities in the $a_{v}$.

(g) Let $R(C) \subset \mathbb{P}\left(R_{d}^{B}\right) \cong \mathbb{P}\left((R / L)_{d}\right)$ be the solution set of these linear equalities and non-equalities. Then 
- $R(C) \subset\left(Z_{1}^{+}(E)\right)_{d}$, i. e. no polynomial in the solution set contains a monomial factor: if $\bar{x}_{i} \mid \bar{f}$ in $R / L$ for some $i \in\{0, \ldots, n\}$ and $[\bar{f}] \in R(C)$ then $x_{i} \mid f_{A}$ for every basis $A$ with $i \in A$. Hence, $\operatorname{Newt}\left(f_{A}\right)$ does not meet all three sides of $\Delta_{d}$ whereas $\operatorname{Newt}\left(C_{A}\right)$ does - in contradiction to $[f] \in R(C)$. So every $[f] \in R(C)$ corresponds to an effective 1-cycle $Y$ of degree $d$.

- $R(C)$ describes exactly the effective 1-cycles tropicalizing to $C$ : this follows from Theorem 4.11, since $\operatorname{Newt}\left(f_{A}\right)=\operatorname{Newt}\left(C_{A}\right)$ means $\operatorname{Newt}\left(\pi_{*}^{A}(Y)\right)=$ $\operatorname{Newt}\left(p_{*}^{A}(C)\right)$ by Construction 4.9, which in turn means $\operatorname{Trop}\left(\pi_{*}^{A}(Y)\right)=p_{*}^{A}(C)$ by Lemma 4.14 .

Hence, $R(C)$ is the realization space $\operatorname{Real}(C)$ of $C$.

In particular, $\operatorname{Real}(C) \subset\left(Z_{1}^{+}(E)\right)_{d} \subset \mathbb{P}\left((R / L)_{d}\right)$ is the complement of a union of hyperplanes in a linear space. Of course, the algorithm can also be used to compute the dimension of $\operatorname{Real}(C)$.

For some purposes, it is interesting to know if a tropical curve in $\operatorname{trop}(E)$ is relatively realizable not only by a positive cycle, but also by an irreducible curve in $E$. Here, a cycle $Y \in Z_{1}(E)$ is called irreducible if it is defined by exactly one irreducible curve in $E$ with multiplicity one. Otherwise, we call this cycle reducible.

To check for this irreducible realizability of a tropical curve $C \subset \operatorname{trop}(E)$, we will consider non-trivial decompositions of $C$ into $D_{1}$ and $D_{2}$, by which we mean two positive tropical cycles $D_{1}, D_{2} \neq 0$ in $Z_{1}^{\text {trop }}(\operatorname{trop}(E))$ such that $D_{1}+D_{2}=C$. Note that in this case $\operatorname{deg}(C)=$ $\operatorname{deg}\left(D_{1}\right)+\operatorname{deg}\left(D_{2}\right)$.

Proposition 4.16. Let $C \subset \operatorname{trop}(E)$ be a tropical curve with realdim $(C)=m$ as in Definition 4.8. Then there is an irreducible cycle in $E$ tropicalizing to $C$ if and only if for every non-trivial decomposition $C=D_{1}+D_{2}$ of $C$ in $Z_{1}^{\text {trop }}(\operatorname{trop}(E))$ with realdim $\left(D_{1}\right)=m_{1}$ and realdim $\left(D_{2}\right)=m_{2}$ we have $m_{1}+m_{2}<m$.

Proof. Let $C=D_{1}+D_{2}$ be a non-trivial decomposition with $\operatorname{deg}\left(D_{1}\right)=d_{1}$ and $\operatorname{deg}\left(D_{2}\right)=$ $d_{2}$, where $d_{1}+d_{2}=d=\operatorname{deg}(C)$. We consider the morphism of varieties

$$
\begin{aligned}
\phi: \mathbb{P}\left((R / L)_{d_{1}}\right) \times \mathbb{P}\left((R / L)_{d_{2}}\right) & \longrightarrow \mathbb{P}\left((R / L)_{d}\right) \\
([\bar{f}],[\bar{g}]) & \longmapsto[\overline{f g}] .
\end{aligned}
$$

As every polynomial of degree $d$ has only finitely many factorizations into two polynomials of degree $d_{1}$ and $d_{2}$ up to scalar multiplication, the map $\phi$ has finite fibers. Hence, the dimension of the image of $\operatorname{Real}\left(D_{1}\right) \times \operatorname{Real}\left(D_{2}\right)$, i. e. the space of cycles in $\operatorname{Real}(C)$ that are composed of two cycles of degrees $d_{1}$ and $d_{2}$, has dimension $m_{1}+m_{2}$. Note that $\phi\left(\operatorname{Real}\left(D_{1}\right) \times \operatorname{Real}\left(D_{2}\right)\right)$ is closed in $\operatorname{Real}(C)$.

If $m=m_{1}+m_{2}$ this implies that $\operatorname{Real}(C)=\phi\left(\operatorname{Real}\left(D_{1}\right) \times \operatorname{Real}\left(D_{2}\right)\right)$, since $\operatorname{Real}(C)$ is irreducible by Algorithm 4.15 as an open subset of a linear space. So in this case $C$ is only realizable by a sum of two cycles realizing $D_{1}$ and $D_{2}$, respectively. 
On the other hand, if $m_{1}+m_{2}<m$ for every non-trivial decomposition of $C$ into $D_{1}+D_{2}$, the space of reducible cycles in $\operatorname{Real}(C)$ is a proper closed subset of $\operatorname{Real}(C)$. Hence, there has to be an irreducible curve in $\operatorname{Real}(C)$, so in this case $C$ is irreducibly realizable.

Using this, Algorithm 4.15 can be extended to check the irreducible relative realizability of a tropical curve in $\operatorname{trop}(E)$ :

Algorithm 4.17. Let $C$ be a realizable tropical curve in $\operatorname{trop}(E)$ and $m$ the realization dimension of $C$ in $E$. For any non-trivial decomposition $C=D_{1}+D_{2} \in Z_{1}^{\text {trop }}(\operatorname{trop}(E))$, where $D_{1}$ and $D_{2}$ are two tropical curves in trop $(E)$, compute the realization dimensions $m_{i}$ of $D_{i}$ in $E$ for $i=1,2$. If there is a decomposition $C=D_{1}+D_{2}$ with $m_{1}+m_{2}=m$, then the tropical curve $C$ is not realizable by an irreducible curve in $E$; if $m_{1}+m_{2}<m$ for all decompositions $C=D_{1}+D_{2}$, then there is an irreducible curve in $E$ tropicalizing to $C$.

Example 4.18 (The Singular library realizationMatroids.lib). The above Algorithms 4.15 and 4.17 deciding (irreducible) relative realizability are implemented in the Singular library realizationMatroids.lib [DGPS, Win12].

Let $C$ be a tropical curve in $\operatorname{trop}(E)=B(M(L))$, and let $P=P(C)$ as in Notation 2.7.

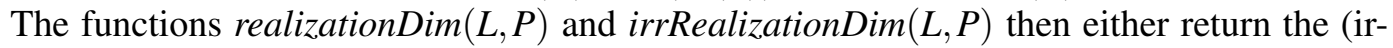
reducible) realization dimension of $C$ in $E$, or -1 if the realization space of $C$ is empty, i. e. if $C$ is not relatively realizable (by an irreducible curve).

Moreover, following Algorithm 4.15 we can explicitly describe the set of polynomials $f$ such that the ideal $L+(f)$ tropicalizes to a given tropical curve $C$ in $\operatorname{trop}(E)$. Correspondingly, the function realizationDimPoly $(L, P)$ returns the realization dimension of $C$ together with a polynomial realizing $C$ in $E$. This function works by checking small integer coefficients for the polynomials and may only be used if the characteristic of $K$ is zero.

The following example shows these functions for the curve $C$ in $L_{2}^{3}=\operatorname{trop}(V(L))$ with $L=\left(x_{0}+x_{1}+x_{2}+x_{3}\right) \subset K\left[x_{0}, \ldots, x_{3}\right]$ and $P(C)=\{(2,2,0,0),(0,0,2,2)\}$.

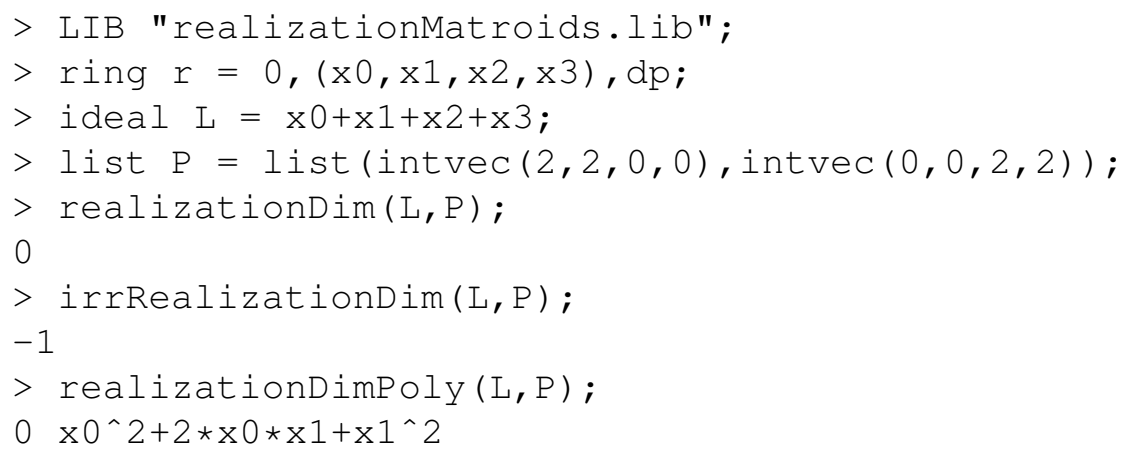

\section{General CRiteria FOR RElative ReAlizabiLity}

5.1. Relative Realizability of Cycles. As in Notation 4.6 let $B(M(L))=\operatorname{trop}(E) \subset$ $\mathbb{R}^{n+1} /\langle\mathbf{1}\rangle$ be the matroid fan obtained by tropicalizing a plane $E$ in a torus $X$. In this 
section we will show that any tropical cycle in $B(M(L))$ is relatively realizable by a cycle in $Z_{1}(E)$, i. e. that the map Trop : $Z_{1}(E) \rightarrow Z_{1}^{\text {trop }}(\operatorname{trop}(E))$ of Definition 4.4 (c) is surjective. In particular, this means that any tropical curve in $B(M(L))$ is relatively realizable by a cycle in $Z_{1}(E)$. To prove this claim, we start by showing that any tropical curve in $B(M(L))$ containing at most one 1-dimensional cone not corresponding to a rank-1 flat is relatively realizable.

Lemma 5.1. Let $C$ be a tropical curve in $\operatorname{trop}(E)$ such that $P(C)=\left\{v, \lambda_{1} v_{F_{1}}, \ldots, \lambda_{r} v_{F_{r}}\right\}$, where $[v] \in \operatorname{trop}(E) \backslash\{0\}, \lambda_{i} \in \mathbb{N}$ for all $i$, and $F_{1}, \ldots, F_{r}$ are rank-1 flats of the matroid $M(L)$ with associated vectors $v_{F_{1}}, \ldots, v_{F_{r}}$ as in Construction 2.5. Then the tropical curve $C$ is realizable in $L$.

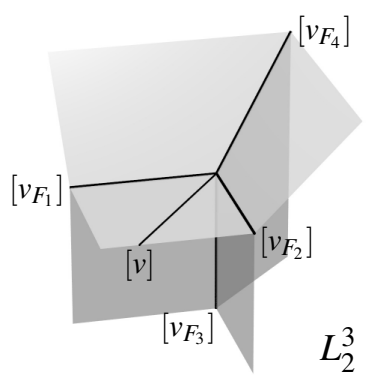

Proof. Since $\operatorname{trop}(E)$ consists of the cones $\sigma_{\mathscr{G}}$, where $\mathscr{G}=\left(G_{1}, G_{2}\right)$ is a chain of flats in $M(L)$, and $[v] \in \operatorname{trop}(E)$, there is a rank-1 flat $G_{1}$ and a rank-2 flat $G_{2}$ such that $[v] \in \sigma_{\mathscr{G}}=\operatorname{cone}\left(\left[v_{G_{1}}\right],\left[v_{G_{2}}\right]\right)$. We denote the elements of the base set of $M=M(L)$ by $0, \ldots, n$. Applying a coordinate permutation, we may assume that there are $0<k_{1}<k_{2} \leq n$ such that $G_{1}=\left\{0, \ldots, k_{1}-1\right\}$ and $G_{2}=\left\{0, \ldots, k_{2}-1\right\}$. Hence, we have

$$
v=a\left(e_{0}+\ldots+e_{k_{1}-1}\right)+b\left(e_{k_{1}}+\ldots+e_{k_{2}-1}\right)
$$

for some $a, b \in \mathbb{N}, b \leq a \leq d:=\operatorname{deg}(C)$, and $(a, b) \neq(0,0)$. With

$$
f=c_{0} x_{0}^{d}+c_{1} x_{k_{1}}^{d}+c_{2} x_{0}^{b} x_{k_{2}}^{d-b}+c_{3} x_{k_{1}}^{a} x_{k_{2}}^{d-a}
$$

for any generic $c=\left(c_{0}, \ldots, c_{3}\right) \in K^{4}$, we claim that $C=\operatorname{Trop}(L+(f))$. To prove this claim, we want to use Theorem 4.11 and therefore show that $\operatorname{Newt}\left(f_{A}\right)=\operatorname{Newt}\left(p_{*}^{A}(C)\right)$ for all bases $A$ of $M$, where $f_{A}$ is as in Construction 4.9.

So let $A=\{i, j, k\}$ a basis of $M$. By Construction 4.9 there are $\lambda_{l}, \mu_{l}, v_{l} \in K$ for $l=i, j, k$ such that

$$
\begin{aligned}
x_{0}-\lambda_{i} x_{i}-\lambda_{j} x_{j}-\lambda_{k} x_{k} & \in L, \\
x_{k_{1}}-\mu_{i} x_{i}-\mu_{j} x_{j}-\mu_{k} x_{k} & \in L, \\
x_{k_{2}}-v_{i} x_{i}-v_{j} x_{j}-v_{k} x_{k} & \in L .
\end{aligned}
$$

Since any two different elements in $\left\{0, \ldots, k_{1}-1\right\}$ are contained in the rank-1 flat $G_{1}$, they are linearly dependent, as are three pairwise different elements in $G_{2}=\left\{0, \ldots, k_{2}-1\right\}$. Hence, we get the following 3 cases.

Case 1: $i \in\left\{0, \ldots, k_{1}-1\right\}, j \in\left\{k_{1}, \ldots, k_{2}-1\right\}, k \in\left\{k_{2}, \ldots, n\right\}$.

As $\{0, i\}$ and $\left\{k_{1}, i, j\right\}$ are linearly dependent, we have $\lambda_{j}=\lambda_{k}=\mu_{k}=0$. It also holds that $\lambda_{i}, \mu_{j}, v_{k} \neq 0$, because $L$ does not contain a monomial and $\left\{0, k_{1}, k_{2}\right\}$ is a basis of $M$. So we have

$$
\begin{gathered}
f_{A}\left(x_{i}, x_{j}, x_{k}\right)=c_{0}\left(\lambda_{i} x_{i}\right)^{d}+c_{1}\left(\mu_{i} x_{i}+\mu_{j} x_{j}\right)^{d}+c_{2}\left(\lambda_{i} x_{i}\right)^{b}\left(v_{i} x_{i}+v_{j} x_{j}+v_{k} x_{k}\right)^{d-b} \\
+c_{3}\left(\mu_{i} x_{i}+\mu_{j} x_{j}\right)^{a}\left(v_{i} x_{i}+v_{j} x_{j}+v_{k} x_{k}\right)^{d-a} .
\end{gathered}
$$


Since $\lambda_{i}, \mu_{j}, v_{k} \neq 0$ and $c \in K^{4}$ is generic, the coefficients of $x_{i}^{d}, x_{j}^{d}, x_{i}^{b} x_{k}^{d-b}$, and $x_{j}^{a} x_{k}^{d-a}$ in this polynomial are non-zero. However, the coefficient of $x_{i}^{m_{i}} x_{j}^{m_{j}} x_{k}^{m_{k}}$ is zero whenever $m_{i}<b$ and $m_{k}>d-a$. As $P\left(p_{*}^{A}(C)\right)=\{(a, b, 0),(d-a, 0,0),(0, d-b, 0),(0,0, d)\}$, we thus have

$$
\operatorname{Newt}\left(f_{A}\right)=\operatorname{conv}((b, 0, d-b),(d, 0,0),(0, d, 0),(0, a, d-a))=\operatorname{Newt}\left(p_{*}^{A}(C)\right) .
$$

Case 2: $i, j \in\left\{k_{1}, \ldots, k_{2}-1\right\}, k \in\left\{k_{2}, \ldots, n\right\}$.

In this case $\{0, i, j\}$ and $\left\{k_{1}, i, j\right\}$ are linearly dependent, and so we have $\lambda_{k}=\mu_{k}=0$. Moreover, we have $\lambda_{i}, \lambda_{j}, \mu_{i}, \mu_{j}, v_{k} \neq 0$ as $\{0, j\},\{0, i\},\left\{k_{1}, j\right\},\left\{k_{1}, i\right\}$ resp. $\left\{k_{2}, i, j\right\}$ are linearly independent. Since

$$
\begin{aligned}
& f_{A}\left(x_{i}, x_{j}, x_{k}\right)= \\
& \quad c_{0}\left(\lambda_{i} x_{i}+\lambda_{j} x_{j}\right)^{d}+c_{1}\left(\mu_{i} x_{i}+\mu_{j} x_{j}\right)^{d}+c_{2}\left(\lambda_{i} x_{i}+\lambda_{j} x_{j}\right)^{b}\left(v_{i} x_{i}+v_{j} x_{j}+v_{k} x_{k}\right)^{d-b} \\
& \quad c_{3}\left(\mu_{i} x_{i}+\mu_{j} x_{j}\right)^{a}\left(v_{i} x_{i}+v_{j} x_{j}+v_{k} x_{k}\right)^{d-a}
\end{aligned}
$$

and $c \in K^{4}$ is generic, we have as in Case 1

$$
\operatorname{Newt}\left(f_{A}\right)=\operatorname{conv}((b, 0, d-b),(d, 0,0),(0, d, 0),(0, b, d-b))=\operatorname{Newt}\left(p_{*}^{A}(C)\right) .
$$

Case 3: $j, k \in\left\{k_{2}, \ldots, n\right\}$.

We have $\left(\lambda_{l}, \mu_{l}\right) \neq(0,0)$ for all $l=i, j, k$ : if for instance $\lambda_{i}=\mu_{i}=0$, then we see by suitable linear combinations of the polynomials $x_{0}-\lambda_{j} x_{j}-\lambda_{k} x_{k} \in L$ and $x_{k_{1}}-\mu_{j} x_{j}-\mu_{k} x_{k} \in L$ that $\left\{0, k_{1}, k\right\}$ and $\left\{0, k_{1}, j\right\}$ are linearly dependent.

Since $c \in K^{4}$ is generic, the coefficient of $x_{i}^{d}$ in $f_{A}$, i. e. the sum $c_{0} \lambda_{i}^{d}+c_{1} \mu_{i}^{d}+c_{2} \lambda_{i}^{b} v_{i}^{d-b}+$ $c_{3} \mu_{i}^{a} v_{i}^{d-a}$, is non-zero, as is the coefficient of $x_{j}^{d}$ and $x_{k}^{d}$. Hence, we have

$$
\operatorname{Newt}\left(f_{A}\right)=\operatorname{conv}((d, 0,0),(0, d, 0),(0,0, d))=\operatorname{Newt}\left(p_{*}^{A}(C)\right) .
$$

So with generic coefficients $c \in K^{4}$, we have $\operatorname{Newt}\left(p_{*}^{A}(C)\right)=\operatorname{Newt}\left(f_{A}\right)$ for all bases $A$ of $M$. Applying Theorem 4.11, we see that $\operatorname{Trop}(L+(f))=C$.

Example 5.2. Let $F_{1}, \ldots, F_{k}$ be the rank-1 flats of $M(L)$. Note that $\sum_{i=1}^{k} v_{F_{i}}=\mathbf{1}$, i. e. these 1-dimensional cones (with multiplicities 1) form a balanced polyhedral fan $D$ in $\operatorname{trop}(E)$ with $P(D)=\left\{v_{F_{1}}, \ldots, v_{F_{k}}\right\}$. By Lemma 5.1, this tropical curve is relatively realizable in $L$.

Using Lemma 5.1, we can now prove the following proposition.

Proposition 5.3. The map Trop : $Z_{1}(E) \rightarrow Z_{1}^{\text {trop }}(\operatorname{trop}(E))$ is surjective.

Proof. Let $C \in Z_{1}^{\text {trop }}(\operatorname{trop}(E))$ be a tropical curve and $v \in P(C)$ not a positive multiple of one of the vectors $v_{F_{1}}, \ldots, v_{F_{k}}$ corresponding to the rank-1 flats $F_{1}, \ldots, F_{k}$ of $M(L)$. As in the proof of Lemma 5.1 we know that $[v] \in \operatorname{cone}\left\{\left[v_{G_{1}}\right],\left[v_{G_{2}}\right]\right\}$, where $\left(G_{1}, G_{2}\right)$ is a chain of flats in $M(L)$. Due to the definition of $v_{G_{2}}$ we know that $v_{G_{2}}=\sum_{G \subset G_{2}} v_{G}$, with the sum taken over all rank-1 flats $G$ contained in $G_{2}$. So we can write $[v]=\sum_{i=1}^{k} a_{i}\left[v_{F_{i}}\right]$ for some $a_{1}, \ldots, a_{k} \in \mathbb{N}$. With $d=\max \left\{a_{1}, \ldots, a_{k}\right\}+1$ there is then a (balanced) tropical curve $D_{v}$ of degree $d$ with $P\left(D_{v}\right)=\left\{v,\left(d-a_{1}\right) v_{F_{1}}, \ldots,\left(d-a_{k}\right) v_{F_{k}}\right\}$. 
By construction, $C-\sum_{v} D_{v}$ is now a balanced cycle in $\operatorname{trop}(E)$ with rays $\left[v_{F_{1}}\right], \ldots,\left[v_{F_{k}}\right]$, where the sum is taken over all $v$ as above. As $v_{F_{1}}, \ldots, v_{F_{k}}$ are linearly independent, this is only possible if $C-\sum_{v} D_{v}=\lambda D$ is a multiple of the tropical curve $D$ of Example 5.2 with $P(D)=\left\{v_{F_{1}}, \ldots, v_{F_{k}}\right\}$. But $D_{v}$ and $D$ are realizable by Lemma 5.1 and Example 5.2. Thus we have $C=\lambda D+\sum_{v} D_{v} \in \operatorname{Trop}\left(Z_{1}(E)\right)$. The map Trop : $Z_{1}(E) \rightarrow Z_{1}^{\text {trop }}(\operatorname{trop}(E))$ is linear and moreover, any cycle in $Z_{1}^{\text {trop }}(\operatorname{trop}(E))$ is $\mathbb{Z}$-linear combination of tropical curves. This shows the surjectivity of Trop.

5.2. Obstructions to Realizability in $L=\left(x_{0}+x_{1}+x_{2}+x_{3}\right)$. In this section, we will use Algorithm 4.15 to give general obstructions to realizability in $L=\left(x_{0}+x_{1}+x_{2}+x_{3}\right) \subset$ $K\left[x_{0}^{ \pm 1}, x_{1}^{ \pm 1}, x_{2}^{ \pm 1}, x_{3}^{ \pm 1}\right]$, where $K$ is any algebraically closed field. More precisely, we will work out conditions on a tropical curve $C$ implying that the Newton polytopes of the tropical push-forwards $p_{*}^{A} C$ of $C$ cannot be the Newton polytopes of the algebraic projections $f_{A}$ of any homogeneous polynomial $f$ whose degree is the degree of the tropical curve $C$. In Theorem 4.11, we have seen that in this case, the tropical curve $C$ is not realizable in $L$. Some of these criteria will depend on the characteristic of $K$, others are independent of the characteristic. Our main results are the Propositions 5.10, 5.15, 5.17, 5.20, and 5.21.

To use dependencies between the Newton polytopes of the push-forwards $p_{*}^{A}(C)$ of a tropical curve $C$ in $L_{2}^{3}$, we will need the coordinates of the vertices of these Newton polytopes. Therefore, we will use an explicit version of the definition of the Newton polytope of a plane tropical curve summarized in the following lemma.

Remark 5.4 (Orientations of $\mathbb{R}^{3} /\langle\mathbf{1}\rangle$ ). For our computation of Newton polytopes it is convenient to choose an orientation of the plane $\mathbb{R}^{3} /\langle\mathbf{1}\rangle$ by calling a basis $\left(\left[v_{1}\right],\left[v_{2}\right]\right)$ positive if $\operatorname{det}\left(v_{1}, v_{2}, \mathbf{1}\right)>0$. For the set $P(C)=\left\{v_{1}, \ldots, v_{r}\right\}$ of a tropical curve $C$ in $\mathbb{R}^{3} /\langle\mathbf{1}\rangle$ we will assume that its vectors $v_{1}, \ldots, v_{r} \in \mathbb{R}^{3}$ are listed in positive order, i. e. that their classes $\left[v_{1}\right], \ldots,\left[v_{r}\right]$ are in positive order with respect to the above orientation, ending with a positive multiple of $e_{0}$ (if present). This means that these vectors $v_{i}=\left(v_{i, 0}, v_{i, 1}, v_{i, 2}\right)$ are sorted like the columns in the following table:

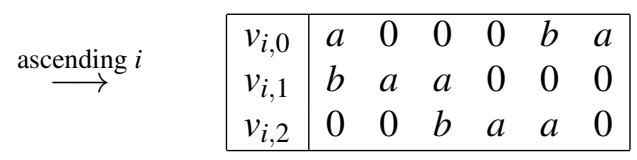

where $a, b \in \mathbb{N}_{>0}$ are arbitrary numbers (depending on $i$ ), and vectors that belong to the same column are sorted according to ascending values of $\frac{b}{a}$.

Lemma 5.5 (Vertices of Newton polytopes). Let $C$ be a plane tropical curve of degree $d$ with $P(C)=\left\{v_{1}, \ldots, v_{r}\right\}$, where the vectors $v_{i}=\left(v_{i, 0}, v_{i, 1}, v_{i, 2}\right)$ for $i=1, \ldots, k$ are sorted in positive order as in Remark 5.4. Then the vertices of $\operatorname{Newt}(C) \subset \Delta_{d}$ are exactly the points

$Q_{k}=(0, m, d-m)+\sum_{i=1}^{k} u_{i} \quad$ with $\quad m=\sum_{i: v_{i, 1} \neq 0} v_{i, 0}$ and $u_{i}=\left(v_{i, 1}-v_{i, 2}, v_{i, 2}-v_{i, 0}, v_{i, 0}-v_{i, 1}\right)$ for $k=0, \ldots, r-1$. 
Proof. As the vectors $\left[v_{1}\right], \ldots,\left[v_{r}\right] \in \mathbb{R}^{3} /\langle\mathbf{1}\rangle$ sum up to zero and are sorted in positive order, the points $\sum_{i=1}^{k}\left[v_{i}\right]$ for $k=0, \ldots, r-1$ are the vertices of a convex polytope. But $Q_{0}, \ldots, Q_{r-1}$ are by construction just the images of these points under an affine isomorphism $\mathbb{R}^{3} /\langle\mathbf{1}\rangle \rightarrow$ $H_{d}:=\left\{\left(w_{0}, w_{1}, w_{2}\right): w_{0}+w_{1}+w_{2}=d\right\}$. Thus they also form the vertices of a convex polytope $\Delta \subset H_{d}$.

To check that $C$ is the weighted inner normal fan of $\Delta$ note first of all that one of the coordinates of each $v_{k}$ is zero by definition of $P(C)$. Hence, for all $k$ we have $\operatorname{gcd}\left(v_{k, 0}, v_{k, 1}, v_{k, 2}\right)=$ $\operatorname{gcd}\left(u_{k, 0}, u_{k, 1}, u_{k, 2}\right)$, and thus $\left[v_{k}\right]$ and $u_{k}=Q_{k}-Q_{k-1}$ have the same integer length. Moreover, it is obvious that $v_{k} \cdot u_{k}=0$. This means that the function $\varphi_{k}: \Delta \rightarrow \mathbb{R}, u \mapsto v_{k} \cdot u$ is constant, and thus extremal, on the side $\overline{Q_{k-1} Q_{k}}$ of $\Delta$. It remains to be shown that it is in fact minimal there. This is obvious if $\Delta$ is 1-dimensional, so let us assume that $\Delta$ is 2-dimensional. In this case balancing requires the oriented angle between $v_{k}$ and $v_{k+1}$ (where we set $\left.v_{r+1}:=v_{1}\right)$ to be in the open interval $(0, \pi)$, which means that $\left(\left[v_{k}\right],\left[v_{k+1}\right]\right)$ is a positive basis of $\mathbb{R}^{3} /\langle\mathbf{1}\rangle$. But then we have

$$
\varphi_{k}\left(Q_{k+1}\right)-\varphi_{k}\left(Q_{k}\right)=v_{k} \cdot u_{k+1}=\operatorname{det}\left(v_{k}, v_{k+1}, \mathbf{1}\right)>0,
$$

which implies minimality as claimed. So, up to translations, $\Delta$ is the Newton polytope of $C$.

Finally, to see that the translation is correct, it suffices by Definition 4.13 to check that $\Delta$ meets the three lines in $H_{d}$ where one of the coordinates is 0 . Obviously, the 0 -th coordinate is 0 for $Q_{0}$. If $k$ corresponds to the last vector in the second column in the table of Remark 5.4, the first coordinate of $Q_{k}$ is

$$
m+\sum_{i=1}^{k}\left(v_{i, 2}-v_{i, 0}\right)=\sum_{i: v_{i, 1} \neq 0} v_{i, 0}-\sum_{i: v_{i, 1} \neq 0} v_{i, 0}=0,
$$

and if $k$ corresponds to the last vector in the fourth column in this table, the second coordinate of $Q_{k}$ is

$$
d-m+\sum_{i=1}^{k}\left(v_{i, 0}-v_{i, 1}\right)=d-\sum_{i: v_{i, 1} \neq 0} v_{i, 0}+\sum_{i: v_{i, 1} \neq 0} v_{i, 0}-\sum_{i=1}^{r} v_{i, 1}=d-m+m-d=0
$$

by Lemma 2.9 .

Notation 5.6 (Notations for Newton polytopes).

(a) To simplify the notations, we denote the projections $p^{A}: \mathbb{R}^{4} /\langle\mathbf{1}\rangle \rightarrow \mathbb{R}^{3} /\langle\mathbf{1}\rangle$ for a basis $A$ of $M(L)$ (see Construction 3.1) by $p^{k}$, where $k \in\{0,1,2,3\}$ is the unique element not contained in $A$. Correspondingly, the polynomial $f_{A}$ of Construction 4.9 is denoted by $f_{k}$.

(b) To work with the Newton polytopes of the push-forwards $p_{*}^{k}(C)$, we will identify the plane $H_{d}=\left\{w \in \mathbb{R}^{3}: w_{0}+w_{1}+w_{2}=d\right\}$ with $\mathbb{R}^{2}$ by choosing the isomorphism $H_{d} \rightarrow \mathbb{R}^{2}, w \mapsto\left(w_{0}, w_{1}\right)$. In other words, from now on the Newton polytopes $\operatorname{Newt}\left(p_{*}^{k}(C)\right)$ and $\operatorname{Newt}\left(f_{k}\right)$ will be considered to be in $\operatorname{conv}((0,0),(0, d),(d, 0)) \subset$ $\mathbb{R}^{2}$ by dropping the last coordinate which is not the $k$-th. 
(c) We will denote the coefficients of $f_{0}$ and $f_{3}$ by $a_{i j}$ and $b_{i j}$, respectively, i.e.

$$
f_{0}\left(x_{1}, x_{2}, x_{3}\right)=\sum_{i=0}^{d} \sum_{j=0}^{d-i} a_{i j} x_{1}^{i} x_{2}^{j} x_{3}^{d-i-j} \quad \text { and } \quad f_{3}\left(x_{0}, x_{1}, x_{2}\right)=\sum_{i=0}^{d} \sum_{j=0}^{d-i} b_{i j} x_{0}^{i} x_{1}^{j} x_{2}^{d-i-j} .
$$

This is illustrated in the following picture:
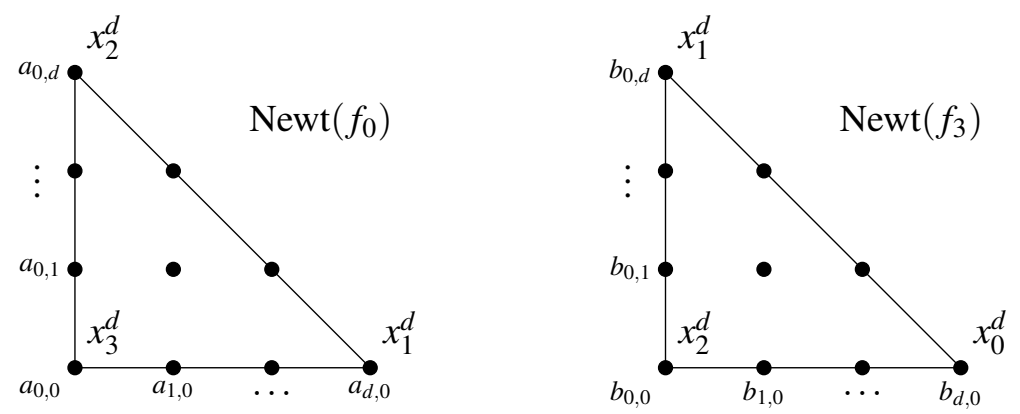

To start with, we want to prove an obstruction to realizability in $L$ equivalent to an obstruction given by Brugallé and Shaw in [BS11]. They proved that if a tropical curve $D$ in $L_{2}^{3}$ is realizable in $L$ by an irreducible curve and $C$ is a tropical curve in $L_{2}^{3}$ such that the tropical intersection product $C \cdot D$ in $L_{2}^{3}$ is negative, then $C$ cannot be realizable by an irreducible curve. In the following, we will give a result which is equivalent to this obstruction in the case where $D$ is one of the classical lines $D_{1}=\operatorname{span}\{[1,1,0,0]\}, D_{2}=\operatorname{span}\{[1,0,1,0]\}$, or $D_{3}=\operatorname{span}\{[1,0,0,1]\}$. However, Brugallé and Shaw always ask for relative realizability by irreducible cycles, while in this paper we allow any positive cycle to realize a given tropical curve. That is why the statements seem to be different at first glance, although in fact the obstruction we obtain is the same.

Remark 5.7 (Correspondence between relative realizability by irreducible and positive cycles). In Proposition 4.16 we have seen that relative realizability by positive cycles may be used to decide relative realizability by irreducible cycles. However, it is also possible to decide relative realizability by positive cycles using the irreducible version of relative realizability: Let $C$ be any tropical curve in $L_{2}^{3}$. To decide whether or not $C$ is relatively realizable by a positive cycle in $Z_{1}(E)$, consider all positive tropical decompositions $C=\sum_{i=1}^{r} C_{i} \in Z_{1}^{\text {trop }}(\operatorname{trop}(E))$. If there is a decomposition $C=\sum_{i=1}^{r} C_{i}$ such that each tropical curve $C_{i}$ is relatively realizable by an irreducible cycle, then $C$ is relatively realizable by a positive cycle. If such a decomposition does not exist, there is no positive cycle in $Z_{1}(E)$ tropicalizing to $C$.

Our algorithm to decide relative realizability by positive cycles is based on projections, and a priori it is not clear how the intersection product of two tropical curves in $L_{2}^{3}$ can be seen in these projections. Therefore, we want to find an interpretation of the intersection product $C \cdot D$ in terms of the Newton polytopes of the push-forwards $p_{*}^{k}(C)$ of $C$ in case $D$ is a classical line. Since the three classical lines are equivalent modulo permutations of the coordinates, we will only state and prove this interpretation for the classical line $D_{1}$. 
Lemma 5.8 (Intersection products with classical lines). Let $C$ be a tropical curve of degree $d$ in $L_{2}^{3}$. Moreover, let $m_{3}$ be the maximum of all $m \in \mathbb{N}$ such that $(i, j) \notin \operatorname{Newt}\left(p_{*}^{3}(C)\right)$ for all $(i, j) \in \mathbb{N}^{2}$ with $i+j<m$. Similarly, let $m_{0}$ be the maximum of all $m \in \mathbb{N}$ such that $(i, j) \notin \operatorname{Newt}\left(p_{*}^{0}(C)\right)$ for all $(i, j) \in \mathbb{N}^{2}$ with $i>d-m$. Then the tropical intersection product of $C$ and $D_{1}=\operatorname{span}\{[1,1,0,0]\}$ can be written as

$$
C \cdot D_{1}=d-m_{0}-m_{3} .
$$

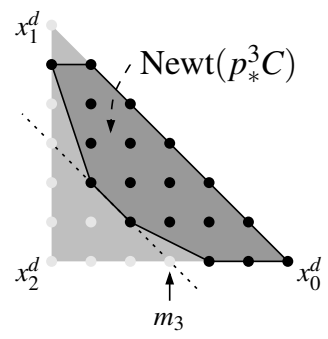

Proof. In Construction 2.12 we have seen that the tropical intersection product of $C$ and $D_{1}$ is given by

$$
C \cdot D_{1}=d-\sum_{(a, b, 0,0) \in P(C)} \min \{a, b\}-\sum_{(0,0, a, b) \in P(C)} \min \{a, b\} .
$$

To prove the claim, we will show that

$$
\sum_{(a, b, 0,0) \in P(C)} \min \{a, b\}=m_{3} .
$$

Analogously, one can then show that

$$
\sum_{(0,0, a, b) \in P(C)} \min \{a, b\}=m_{0} .
$$

Let $\left(a_{1}, b_{1}, 0,0\right), \ldots,\left(a_{r}, b_{r}, 0,0\right)$ be all elements in $P(C)$ whose first and second coordinate are both non-zero, sorted in such a way that

$$
\frac{b_{k}}{a_{k}}<\frac{b_{k+1}}{a_{k+1}} \quad \text { for all } k=1, \ldots, r-1 .
$$

By Remark 5.4 this corresponds to the first vectors of a positive ordering of the rays of $p_{*}^{3}(C)$. Hence, by Lemma 5.5 the point $Q_{k}=\left(\sum_{l=1}^{k} b_{l}, \sum_{l=k+1}^{r} a_{l}\right)$ is a vertex of $\operatorname{Newt}\left(p_{*}^{3}(C)\right)$ for all $k=0, \ldots, r$. We then have

$$
\operatorname{Newt}\left(p_{*}^{3}(C)\right) \subset \operatorname{conv}\left(\left\{Q_{k}: k=0, \ldots, r\right\} \cup\{(0, d),(d, 0)\}\right) .
$$

Since $\sum_{l=1}^{k} b_{l}+\sum_{l=k+1}^{r} a_{l} \geq \sum_{l=1}^{r} \min \left\{a_{l}, b_{l}\right\}$ for every $k$, we see that $i+j \geq \sum_{l=1}^{r} \min \left\{a_{l}, b_{l}\right\}$ for all $(i, j) \in \operatorname{Newt}\left(p_{*}^{3}(C)\right)$. On the other hand, the points are in such an ordering that there is a unique $0 \leq k \leq r$ with $\frac{b_{l}}{a_{l}} \leq 1$ for all $l \leq k$ and $\frac{b_{l}}{a_{l}}>1$ for all $l>k$. In other words, $b_{l} \leq a_{l}$ for all $l \leq k$ and $b_{l}>a_{l}$ for all $l>k$. So for this particular $k$ we get

$$
\sum_{l=1}^{k} b_{l}+\sum_{l=k+1}^{r} a_{l}=\sum_{l=1}^{k} \min \left\{a_{l}, b_{l}\right\}+\sum_{l=k+1}^{r} \min \left\{a_{l}, b_{l}\right\}=\sum_{l=1}^{r} \min \left\{a_{l}, b_{l}\right\} .
$$

Hence, we have $m_{3}=\sum_{l=1}^{r} \min \left\{a_{l}, b_{l}\right\}$.

Using this interpretation of the intersection product in terms of the Newton polytopes of the push-forwards $p_{*}^{3}(C)$ and $p_{*}^{0}(C)$, we are aiming to prove the first obstruction to relative realizability, which will imply the obstruction of Brugallé and Shaw in the special case 
when the realizable curve is the classical line $D_{1}=\operatorname{span}\{[1,1,0,0]\}$. To do so, we need the following lemma.

Lemma 5.9. Let $C$ be a tropical curve of degree $d$ in $L_{2}^{3}$, and let $m_{0}$ and $m_{3}$ be as in Lemma 5.8. Moreover, set

$n_{0}=\left|\left\{\left(d-m_{0}, j\right) \in \mathbb{N}^{2}: j \leq m_{0},\left(m_{0}, j\right) \notin \operatorname{Newt}\left(p_{*}^{0}(C)\right)\right\}\right| \quad$ and

$n_{3}=\left|\left\{(i, j) \in \mathbb{N}^{2}: i+j=m_{3},(i, j) \notin \operatorname{Newt}\left(p_{*}^{3}(C)\right)\right\}\right|$.

If $n_{0}>d-m_{3}$ or $n_{3}>d-m_{0}$, then $C$ is not relatively realizable in $L$.

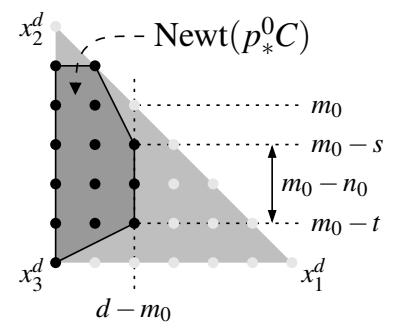

Proof. It suffices by symmetry to show that $C$ is not relatively realizable if $n_{0}>d-m_{3}$. So assume to the contrary that this inequality holds and $C=\operatorname{Trop}(L+(f))$ for a homogeneous polynomial $f \in K\left[x_{0}, x_{1}, x_{2}, x_{3}\right]$ of degree $d$.

By definition of $m_{0}$ the polynomial $f_{0}$ of Notation 5.6 (c) (whose Newton polytope is shown in the picture above) has $x_{1}$-degree $d-m_{0}$. Hence, the (inhomogeneous) polynomial

$$
g\left(x_{3}\right):=\frac{\partial^{d-m_{0}} f_{0}}{\partial x_{1}^{d-m_{0}}}\left(x_{1}, 1, x_{3}\right)
$$

is independent of $x_{1}$; its coefficients correspond to the vertical dotted line in the picture. By definition of $n_{0}$ there are thus $0 \leq s \leq t \leq m_{0}$ with $t-s=m_{0}-n_{0}$ such that $g$ contains only terms of degrees from $s$ to $t$, with non-zero coefficients in degrees $s$ and $t$. Hence, $g$ has degree $t$ and a zero of order $s$ at 0 .

On the other hand, the polynomial $f_{3}\left(x_{0}, x_{1}, x_{2}\right)=f_{0}\left(x_{1}, x_{2},-x_{0}-x_{1}-x_{2}\right)$ is of $x_{2}$-degree $d-m_{3}$ by definition of $m_{3}$. Substituting $-x_{3}-x_{1}$ for $x_{0}$ in $f_{3}$, we thus get another polynomial $f_{0}\left(x_{1}, x_{2}, x_{3}-x_{2}\right)$ whose $x_{2}$-degree is at most $d-m_{3}$. At the same time this polynomial has $x_{1}$-degree at most $d-m_{0}$ just as $f_{0}\left(x_{1}, x_{2}, x_{3}\right)$. Consequently, it does not contain any term with a power of $x_{3}$ less than $m_{0}+m_{3}-d$, i. e. $x_{3}^{m_{0}+m_{3}-d}$ divides $f_{0}\left(x_{1}, x_{2}, x_{3}-x_{2}\right)$. Setting $x_{2}=1$ and replacing $x_{3}$ by $x_{3}+1$ we thus see that $\left(x_{3}+1\right)^{m_{0}+m_{3}-d}$ divides $f_{0}\left(x_{1}, 1, x_{3}\right)$, and hence, also $g$. In other words, $g$ has a zero of order at least $m_{0}+m_{3}-d$ at -1 .

Altogether, we have now seen that the polynomial $g$ has degree $t$ but zeroes of total order $s+\left(m_{0}+m_{3}-d\right)=\left(t+n_{0}-m_{0}\right)+m_{0}+m_{3}-d>\left(t+d-m_{3}-m_{0}\right)+m_{0}+m_{3}-d=t$, which is a contradiction.

Proposition 5.10. Let $C$ be a tropical curve in $L_{2}^{3}$, and let $D$ be the classical line $D=$ $\operatorname{span}\{[1,1,0,0]\}$. Let $k_{1}, k_{2} \in \mathbb{N}_{\geq 0}$ be the multiplicities of the rays $(1,1,0,0)$ and $(0,0,1,1)$ in $C$, respectively, and set $k=\min \left(k_{1}, k_{2}\right)$.

If $C \cdot D<-k$, then the tropical curve $C$ is not realizable in $L$.

Proof. Assume without loss of generality that $k=k_{2}$. Note that $k_{2}$ is just the length $m_{0}-n_{0}$ of the right vertical edge of the Newton polytope $\operatorname{Newt}\left(p_{0}^{*} C\right)$ as in Lemma 5.9. Hence,

$$
n_{0}=m_{0}-k_{2}=m_{0}-k>m_{0}+C \cdot D=d-m_{3},
$$


with the last equation following from Lemma 5.8. So $C$ is not realizable in $L$ by Lemma 5.9 .

Example 5.11. Let $C$ in $L_{2}^{3}$ be the tropical curve with

$$
P(C)=\{(2,2,0,0),(0,0,2,1),(0,0,0,1)\} .
$$

Then we have $C \cdot D_{1}=-1$ by Construction 2.12, so applying Proposition 5.10 we see that the tropical curve $C$ is not realizable in $L$.

Example 5.12. Consider the tropical curve $C$ in $L_{2}^{3}$ with

$$
P(C)=\{(2,1,0,0),(1,1,0,0),(0,1,1,0),(0,0,1,2),(0,0,1,1)\} .
$$

We have $C \cdot D_{1}=-1$, but $C$ is relatively realizable by $f=-x_{1}^{2} x_{2}-2 x_{1} x_{2}^{2}-x_{2}^{3}+x_{1}^{2} x_{3}+$ $x_{1} x_{2} x_{3}=\left(x_{1}+x_{2}\right) \cdot\left(-x_{1} x_{2}-x_{2}^{2}+x_{1} x_{3}\right)$. In this example, we see that the inequality in Proposition 5.10 is sharp. Furthermore, it is not a coincidence that the polynomial $f$ realizing $C$ is reducible. Either by using our algorithm or by applying the obstruction by Brugalle and Shaw, we see that $C$ is not relatively realizable by an irreducible cycle in $Z_{1}(E)$.

Remark 5.13. We claimed that Proposition 5.10 and the obstruction given by Brugalle and Shaw are in fact the same. Example 5.12 shows that in case of relative realizability by positive cycles, the given inequality in Proposition 5.10 is sharp. Hence, no stronger obstruction is possible related to intersection products with classical lines. On the other hand, the obstruction by Brugallé and Shaw implies Proposition 5.10: Given a tropical curve $C$ in $L_{2}^{3}$ fulfilling the conditions of Proposition 5.10, we consider any positive decomposition $C=\sum_{i=1}^{r} C_{i}+m \cdot D_{1}$, where $m \leq k$ and $C_{i} \neq D_{1}$ for all $i=1, \ldots, r$. Since $C \cdot D_{1}<-k$ for $k$ as in Proposition 5.10, we know there is $i \in\{1, \ldots, r\}$ with $C_{i} \neq D_{1}$ and $C_{i} \cdot D_{1}<0$. Hence, in any decomposition of $C$, there is a component which is not realizable by an irreducible cycle and thus, the tropical curve $C$ is not realizable by a positive cycle either.

In the proof of Lemma 5.9 and Proposition 5.10, we have seen that the non-realizability of the tropical curve $C$ follows from dependencies between the Newton polytopes $\operatorname{Newt}\left(f_{k}\right)$. In the rest of this paper it will always be the idea to find suitable dependencies between these Newton polytopes to prove criteria for realizability. However, we first have to know how to translate conditions on $C$ to the Newton polytopes $\operatorname{Newt}\left(p_{*}^{k}(C)\right)$.

Lemma 5.14. Let $\{i, j, k, l\}=\{0,1,2,3\}$, and let $C$ be a tropical curve of degree $d$ in $L_{2}^{3}$ which does not intersect the relative interior of cone $\left(\left[e_{i}\right],\left[e_{j}\right]\right)$. Then

(a) the lattice point corresponding to $x_{k}^{d}$ is contained in $\operatorname{Newt}\left(p_{*}^{l}(C)\right)$, and

(b) the side of $\operatorname{Newt}\left(p_{*}^{j}(C)\right)$ opposite the vertex corresponding to $x_{i}^{d}$ has lattice length $m_{C}\left(e_{i}\right)$.

Proof. By applying a coordinate permutation we may assume that $(i, j, k, l)=(0,1,2,3)$, i. e. $C$ does not intersect the relative interior of cone $\left(\left[e_{0}\right],\left[e_{1}\right]\right)$. Then:

(a) The curve $p_{*}^{3}(C)$ does not intersect cone $\left(\left[e_{0}\right],\left[e_{1}\right]\right)$ either, so with the notation of Lemma 5.5 it follows immediately that $m=0$ and thus $Q_{0}=(0,0) \in \operatorname{Newt}\left(p_{*}^{3}(C)\right)$. 
(b) The ray cone $\left(\left[e_{0}\right]\right)$ (if present) is the only one in $C$ projecting to cone $\left(\left[e_{0}\right]\right)$ under $p^{1}$, so we have $m_{C}\left(e_{0}\right)=m_{p_{*}^{1}(C)}\left(e_{0}\right)$. By definition of the Newton polytope, this is just the lattice length of the edge of $\operatorname{Newt}\left(p_{*}^{1}(C)\right)$ opposite the vertex corresponding to $x_{0}^{d}$.

We are now ready to prove the first new obstruction to realizability. This obstruction considers tropical curves in $L_{2}^{3}$ which are completely contained in three 2-dimensional cones intersecting in a common face. In the following picture, they are the shaded cones.

Proposition 5.15. Let $i, j, k, l \in \mathbb{N}$ with $\{i, j, k, l\}=\{0,1,2,3\}$, and let $C \subset L_{2}^{3}$ be a tropical curve such that

$$
C \subset \operatorname{cone}\left(\left[e_{i}\right],\left[e_{j}\right]\right) \cup \operatorname{cone}\left(\left[e_{i}\right],\left[e_{k}\right]\right) \cup \operatorname{cone}\left(\left[e_{i}\right],\left[e_{l}\right]\right)
$$

and $m_{C}\left(e_{j}\right)=m_{C}\left(e_{k}\right)=0$. Then $C$ can only be realizable in $L$ if $m_{C}\left(e_{l}\right) \neq 1$.

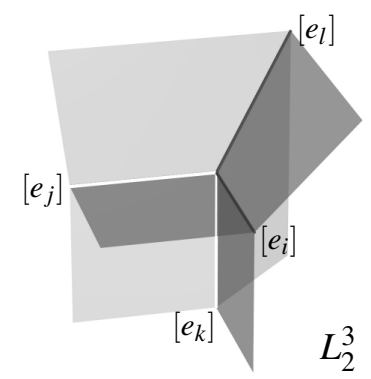

Proof. By applying a suitable coordinate permutation, we may assume that $i=1$ and $l=3$. Assume that $C$ is realizable in $L$ with $m_{C}\left(e_{3}\right)=1$. Let $f \in K\left[x_{0}, x_{1}, x_{2}, x_{3}\right]$ be homogeneous of degree $d=\operatorname{deg}(C)$ such that $C=\operatorname{Trop}(L+(f))$.

First we want to have a closer look at the Newton polytope $\operatorname{Newt}\left(f_{3}\right)=\operatorname{Newt}\left(p_{*}^{3}(C)\right)$. Since $C$ does not intersect cone $\left(\left[e_{0}\right],\left[e_{2}\right]\right)$, we get by Lemma 5.14 (a) that the lattice point $(0, d)$ corresponding to $x_{1}^{d}$ is contained in $\operatorname{Newt}\left(p_{*}^{3}(C)\right)$. Moreover, $C$ does not intersect the relative interiors of cone $\left(\left[e_{0}\right],\left[e_{3}\right]\right)$ and cone $\left(\left[e_{2}\right],\left[e_{3}\right]\right)$. So $m_{C}\left(e_{0}\right)=m_{C}\left(e_{2}\right)=0$ implies by Lemma 5.14 (b) that $\operatorname{Newt}\left(p_{*}^{3}(C)\right)$ meets both $\operatorname{conv}((0,0),(0, d))$ and $\operatorname{conv}((0, d),(d, 0))$ in a single point, which must therefore be the above point $(0, d)$. Hence, the other points on these two lines are not contained in this Newton polytope, i. e. using Notation 5.6 (c) we have

$$
b_{0, d-r}=b_{r, d-r}=0 \quad \text { for all } r=1, \ldots, d .
$$

Now consider $\operatorname{Newt}\left(f_{0}\right)=\operatorname{Newt}\left(p_{*}^{0}(C)\right)$ : since $C$ does not intersect the relative interior of $\operatorname{cone}\left(\left[e_{2}\right],\left[e_{3}\right]\right)$, we know by Lemma 5.14 (a) that the lattice point $(d, 0)$ corresponding to $x_{1}^{d}$ is contained in $\operatorname{Newt}\left(p_{*}^{0}(C)\right)$. Moreover, this Newton polytope intersects conv $((d, 0),(0, d))$ with lattice length $m_{C}\left(e_{3}\right)=1$ by Lemma 5.14 (b). Since $\operatorname{Newt}\left(p_{*}^{0}(C)\right)$ is convex, this means that $(d-r, r) \in \operatorname{Newt}\left(p_{*}^{0}(C)\right) \cap \mathbb{N}^{2}$ if and only if $r \in\{0,1\}$. Hence, we have

$$
\begin{aligned}
a_{d-r, r}=0 & \text { for all } r=2, \ldots, d, \\
a_{d-1,1} \neq 0 . &
\end{aligned}
$$

But we know that $f_{0}\left(x_{1}, x_{2}, x_{3}\right)=f_{3}\left(-x_{1}-x_{2}-x_{3}, x_{1}, x_{2}\right)$, so in particular it holds that

$$
f_{0}(1,-1,0)-f_{0}(1,0,0)=f_{3}(0,1,-1)-f_{3}(-1,1,0) .
$$


Considering the coefficients of $f_{0}$ and $f_{3}$, this equality results in

$$
\sum_{r=1}^{d}(-1)^{r} a_{d-r, r}=\sum_{r=1}^{d}(-1)^{r} b_{0, d-r}-\sum_{r=1}^{d}(-1)^{r} b_{r, d-r} .
$$

This is a contradiction, since the above results show that of the terms in this equation exactly $a_{d-1,1}$ is non-zero. Hence, $C$ can only be realizable in $L$ if $m_{C}\left(e_{3}\right) \neq 1$.

Example 5.16. Let $C$ in $L_{2}^{3}$ be the tropical curve with

$$
P(C)=\{(4,1,0,0),(0,1,4,0),(0,2,0,3),(0,0,0,1)\} .
$$

Then $C \subset \operatorname{cone}\left(\left[e_{0}\right],\left[e_{1}\right]\right) \cup \operatorname{cone}\left(\left[e_{1}\right],\left[e_{2}\right]\right) \cup \operatorname{cone}\left(\left[e_{1}\right],\left[e_{3}\right]\right)$ with $m_{C}\left(e_{0}\right)=m_{C}\left(e_{2}\right)=0$ and $m_{C}\left(e_{3}\right)=1$. Applying Proposition 5.15, we see that $C$ is not realizable in $L$.

A similar obstruction can be proved if the tropical curve is completely contained in two opposite faces of $L_{2}^{3}$.

Proposition 5.17. Let $i, j, k, l \in \mathbb{N}$ such that $\{i, j, k, l\}=$ $\{0,1,2,3\}$, and let $C \subset L_{2}^{3}$ be a tropical curve such that

$$
C \subset \operatorname{cone}\left(\left[e_{i}\right],\left[e_{j}\right]\right) \cup \operatorname{cone}\left(\left[e_{k}\right],\left[e_{l}\right]\right)
$$

and $m_{C}\left(e_{i}\right)=m_{C}\left(e_{j}\right)=m_{C}\left(e_{k}\right)=0$. Then $C$ can only be realizable in $L$ if $m_{C}\left(e_{l}\right) \neq 1$.

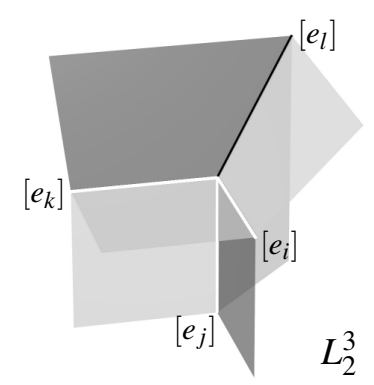

Proof. We may assume that $(i, j, k, l)=(0,1,2,3)$. Assume moreover that $C$ is realizable in $L$ with $m_{C}\left(e_{3}\right)=1$. Let $f \in K\left[x_{0}, x_{1}, x_{2}, x_{3}\right]$ be homogeneous of degree $d=\operatorname{deg}(C)$ such that $C=\operatorname{Trop}(L+(f))$.

We first consider $\operatorname{Newt}\left(f_{3}\right)=\operatorname{Newt}\left(p_{*}^{3}(C)\right)$. As $C$ intersects neither cone $\left(\left[e_{0}\right],\left[e_{2}\right]\right)$ nor $\operatorname{cone}\left(\left[e_{1}\right],\left[e_{2}\right]\right)$, we see by Lemma 5.14 (a) that the lattice points $(d, 0)$ and $(0, d)$ corresponding to $x_{0}^{d}$ and $x_{1}^{d}$, respectively, are contained in this Newton polytope. Moreover, part (b) of this lemma shows that $\operatorname{Newt}\left(p_{*}^{3}(C)\right)$ intersects both $\operatorname{conv}((0,0),(d, 0))$ and $\operatorname{conv}((0,0),(0, d))$ with lattice length 0 , hence, in the points $(d, 0)$ and $(0, d)$, respectively. So with Notation 5.6 (c) we have

$$
b_{r, 0}=b_{0, r}=0 \quad \text { for all } r=0, \ldots, d-1 .
$$

We now have a closer look at $\operatorname{Newt}\left(f_{0}\right)=\operatorname{Newt}\left(p_{*}^{0}(C)\right)$. As $C$ does not intersect the relative interiors of cone $\left(\left[e_{1}\right],\left[e_{2}\right]\right)$ and cone $\left(\left[e_{1}\right],\left[e_{3}\right]\right)$, we have $(0,0),(0, d) \in \operatorname{Newt}\left(p_{*}^{0}(C)\right)$. Moreover, we see that this Newton polytope meets $\operatorname{conv}((0,0),(d, 0))$ in a single point and $\operatorname{conv}((d, 0),(0, d))$ with lattice length 1 . Hence,

$$
\begin{aligned}
a_{r, 0}=0 \quad \text { for all } r=1, \ldots, d, \\
a_{d-r, r}=0 \quad \text { for all } r=0, \ldots, d-2, \\
a_{1, d-1} \neq 0
\end{aligned}
$$


Since $f_{0}\left(x_{1}, x_{2}, x_{3}\right)=f_{3}\left(-x_{1}-x_{2}-x_{3}, x_{1}, x_{2}\right)$, we have

$$
\begin{aligned}
& f_{0}(1,-1,0)-f_{0}(0,-1,0)-f_{0}(1,0,-1)+f_{0}(0,0,-1) \\
= & f_{3}(0,1,-1)-f_{3}(1,0,-1)-f_{3}(0,1,0)+f_{3}(1,0,0),
\end{aligned}
$$

so considering the coefficients of $f_{0}$ and $f_{3}$ we obtain the contradiction

$$
\sum_{r=0}^{d-1}(-1)^{r} a_{d-r, r}-\sum_{r=1}^{d}(-1)^{d-r} a_{r, 0}=\sum_{r=0}^{d-1}(-1)^{d-r} b_{0, r}-\sum_{r=0}^{d-1}(-1)^{d-r} b_{r, 0}
$$

as all terms except $a_{1, d-1}$ are zero in this equation. Hence, $C$ can only be realizable in $L$ if $m_{C}\left(e_{3}\right) \neq 1$.

Example 5.18. Let $C$ in $L_{2}^{3}$ be the tropical curve with

$$
P(C)=\{(3,1,0,0),(1,3,0,0),(0,0,3,1),(0,0,1,2),(0,0,0,1)\} .
$$

Then $C \subset \operatorname{cone}\left(\left[e_{0}\right],\left[e_{1}\right]\right) \cup \operatorname{cone}\left(\left[e_{2}\right],\left[e_{3}\right]\right)$ with $m_{C}\left(e_{i}\right)=0$ for $i=0,1,2$ and $m_{C}\left(e_{3}\right)=1$, so $C$ is not realizable in $L$ by Proposition 5.17.

Our next obstruction to realizability, Proposition 5.20 below, depends on the characteristic of $K$, since the following preparatory lemma does so.

Lemma 5.19. Let $C \subset L_{2}^{3}$ be a tropical curve of degree $d$ in $L_{2}^{3}$, and assume that $\operatorname{char}(K)=0$ or $\operatorname{char}(K) \geq d$. Moreover, let $c \in\{1, \ldots, d-1\}$ and set

$$
\begin{gathered}
A=\{(d-k, k): 0 \leq k \leq d, k \neq c\} \\
\cup\{(d-1-k, k): 0 \leq k \leq d-1\} \quad \text { and } \\
B=\{(0, d-k): 0 \leq k \leq d, k \neq c\},
\end{gathered}
$$

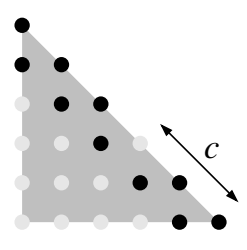

A

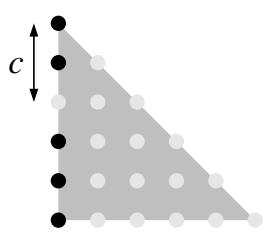

$B$

as indicated with the black dots in the picture on the right.

If $A \cap \operatorname{Newt}\left(p_{*}^{0}(C)\right)=\emptyset$ and $\left|B \cap \operatorname{Newt}\left(p_{*}^{3}(C)\right)\right|=1$, then $C$ is not realizable in $L$.

Proof. Assume that $C$ is realizable in $L$, and let $f \in K\left[x_{0}, x_{1}, x_{2}, x_{3}\right]$ be homogeneous of degree $d$ with $C=\operatorname{Trop}(L+(f))$. As $f_{0}\left(x_{1}, x_{2}, x_{3}\right)=f_{3}\left(-x_{1}-x_{2}-x_{3}, x_{1}, x_{2}\right)$, we have

$$
\text { and } \begin{aligned}
\frac{\partial f_{0}}{\partial x_{1}}\left(x_{1}, x_{2}, x_{3}\right) & =\left(\frac{\partial f_{3}}{\partial x_{1}}-\frac{\partial f_{3}}{\partial x_{0}}\right)\left(-x_{1}-x_{2}-x_{3}, x_{1}, x_{2}\right) \\
\text { a } & \left.x_{2}, x_{3}\right)=-\frac{\partial f_{3}}{\partial x_{0}}\left(-x_{1}-x_{2}-x_{3}, x_{1}, x_{2}\right) .
\end{aligned}
$$

Thus, we get the equality

$$
\begin{aligned}
& \frac{\partial f_{0}}{\partial x_{1}}(1,-1,0)-\frac{\partial f_{0}}{\partial x_{3}}(1,-1,0)-(d-c) \cdot f_{0}(1,-1,0) \\
= & \frac{\partial f_{3}}{\partial x_{1}}(0,1,-1)-(d-c) \cdot f_{3}(0,1,-1) .
\end{aligned}
$$


Considering the coefficients of $f_{0}$ and $f_{3}$ as in Notation 5.6 (c), the above equality reads

$$
\sum_{k=0}^{d}(-1)^{k}(c-k) a_{d-k, k}-\sum_{k=0}^{d-1}(-1)^{k} a_{d-1-k, k}=\sum_{k=0}^{d}(-1)^{k}(c-k) b_{0, d-k} .
$$

By assumption this simplifies to

$$
(c-t) b_{0, d-t}=0,
$$

where $t \in\{0, \ldots, d\}$ with $t \neq c$ is such that $B \cap \operatorname{Newt}\left(p_{*}^{3}(C)\right)=\{(0, d-t)\}$. But $|c-t|<d$, and hence, $c-t \neq 0 \in K$ by our assumption on $\operatorname{char}(K)$. This means that $b_{0, d-t}=0$. But due to the form of $B$ and the fact that the Newton polytope $\operatorname{Newt}\left(p_{*}^{3}(C)\right)$ is convex this implies that $(0, d-t) \notin \operatorname{Newt}\left(p_{*}^{3}(C)\right)$, in contradiction to our assumption.

Proposition 5.20. Let $C \subset L_{2}^{3}$ be a tropical curve of degree $d$, and assume that $\operatorname{char}(K)=0$ or $\operatorname{char}(K) \geq d$. Moreover, let $\{i, j, k, l\}=\{0,1,2,3\}$ such that $C$ intersects neither cone $\left(\left[e_{i}\right],\left[e_{j}\right]\right)$, nor cone $\left(\left[e_{i}\right],\left[e_{i}+e_{k}\right]\right)$, nor cone $\left(\left[e_{i}\right],\left[e_{i}+e_{l}\right]\right)$. If

$$
c_{1}:=\sum_{a e_{j}+b e_{k} \in P(C)} a \neq \sum_{a e_{i}+b e_{k} \in P(C)} a=: c_{2},
$$

and $0<c_{2}<d$, then $C$ is not realizable in $L$.

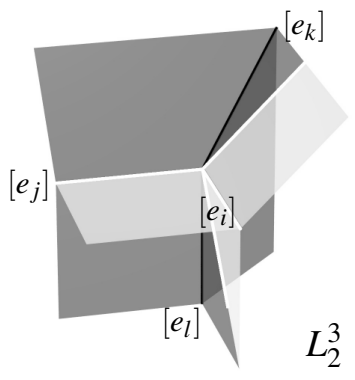

Proof. We may assume that $(i, j, k, l)=(3,0,2,1)$. The idea of the following proof is to show that Lemma 5.19 can be applied with $c:=c_{2}$. So let us determine the intersection of the Newton polytopes $\operatorname{Newt}\left(p_{*}^{0}(C)\right)$ and $\operatorname{Newt}\left(p_{*}^{3}(C)\right)$ with the sets $A$ and $B$ of this lemma, respectively.

As $C$ does not intersect cone $\left(\left[e_{0}\right],\left[e_{3}\right]\right)$, we know by Lemma 5.14 (b) that $\operatorname{Newt}\left(p_{*}^{3}(C)\right)$ meets $\operatorname{conv}((0,0),(0, d))$ in a single point. Lemma 5.5 tells us that this point is $Q_{0}=(0, m)$ with

$$
m=\sum_{a e_{0}+b e_{1} \in P(C)} a=d-\sum_{a e_{0}+b e_{2} \in P(C)} a=d-c_{1},
$$

where the second equality follows from Lemma 2.9 together with the fact that $C$ does not intersect cone $\left(\left[e_{0}\right],\left[e_{3}\right]\right)$. Since $c_{1} \neq c_{2}=c$ by assumption, this implies that $B \cap$ $\operatorname{Newt}\left(p_{*}^{3}(C)\right)=\left\{\left(0, d-c_{1}\right)\right\}$, i. e. $\left|B \cap \operatorname{Newt}\left(p_{*}^{3}(C)\right)\right|=1$.

In the same way, we see that $\operatorname{Newt}\left(p_{*}^{0}(C)\right)$ intersects $\operatorname{conv}((d, 0),(0, d))$ in the single point $\left(d-c_{2}, c_{2}\right)=(d-c, c)$. We will now prove that $(d-1-k, k) \notin \operatorname{Newt}\left(p_{*}^{0}(C)\right)$ for all $k=$ $0, \ldots, d-1$. As $C$ is of degree $d$ and the Newton polytope is convex, it suffices to show that $(d-c-1, c) \notin \operatorname{Newt}\left(p_{*}^{0}(C)\right)$ and $(d-c, c-1) \notin \operatorname{Newt}\left(p_{*}^{0}(C)\right)$. Let us assume first that $(d-c-1, c) \in \operatorname{Newt}\left(p_{*}^{0}(C)\right)$. Since $(d-(c-1), c-1) \notin \operatorname{Newt}\left(p_{*}^{0}(C)\right)$ and $\operatorname{Newt}\left(p_{*}^{0}(C)\right)$ touches $\operatorname{conv}((0,0),(0, d))$, there is an edge of $\operatorname{Newt}\left(p_{*}^{0}(C)\right)$ from $(d-c, c)$ to a point $(a, b)$ with $b \geq c$ and $a+b<d$. So the Newton polytope $\operatorname{Newt}\left(p_{*}^{0}(C)\right)$ contains an edge with directional vector $(a+c-d, b-c)$. This means that

$$
\operatorname{cone}([c-b, a+c-d, 0])=\operatorname{cone}([d-a-b, 0, d-a-c])
$$

is contained in $p_{*}^{0}(C)$, and thus

$$
\sigma=\operatorname{cone}([0, d-a-b, 0, d-a-c])
$$


is contained in $C$. But since $b \geq c$ we have $\sigma \subset \operatorname{cone}\left(\left[e_{3}\right],\left[e_{3}+e_{1}\right]\right)$, in contradiction to the assumption. Similarly, one shows that $(d-c, c-1) \notin \operatorname{Newt}\left(p_{*}^{0}(C)\right)$, because otherwise $C$ would contain a 1 -dimensional cone in cone $\left(\left[e_{3}\right],\left[e_{3}+e_{2}\right]\right)$. Altogether this means that $A \cap \operatorname{Newt}\left(p_{*}^{0}(C)\right)=\emptyset$.

The statement of the proposition now follows from Lemma 5.19.

In [BK11], Bogart and Katz gave another obstruction to realizability in the case char $(K)=$ 0: If a tropical curve $C$ in $L_{2}^{3}$ is also contained in a classical plane $H$ (i. e. in a tropical surface which is at the same time a vector subspace of $\mathbb{R}^{4} /\langle\mathbf{1}\rangle$ ), they proved that $C$ can only be realizable if it contains a classical line or is a multiple of the tropical intersection $L_{2}^{3} \cdot H$ (see Lemma 2.11 ). We will now reprove this obstruction by applying Proposition 5.20 , thereby showing that the statement is also true if $\operatorname{char}(K) \geq \operatorname{deg}(C)$.

Proposition 5.21. [BK11, Proposition 1.3] Let $C \subset L_{2}^{3}$ be a tropical curve of degree $d$ contained in a classical plane $H$, and assume that $\operatorname{char}(K)=0$ or $\operatorname{char}(K) \geq d$. If $C$ is realizable in $L$, then one of the following must hold:

(a) There is $m \in \mathbb{Q}_{>0}$ such that $P(C)=\left\{m \cdot v: v \in P\left(L_{2}^{3} \cdot H\right)\right\}$, in which case we call $C$ a multiple of the tropical intersection $L_{2}^{3} \cdot H$.

(b) The tropical intersection $L_{2}^{3} \cdot H$ contains a classical line.

Proof. As $H$ is a tropical variety, there is $a \in \mathbb{Z}^{4} \backslash\{0\}$ with $a_{0}+a_{1}+a_{2}+a_{3}=0$ such that $H=\left\{[x] \in \mathbb{R}^{4} /\langle\mathbf{1}\rangle: a \cdot x=0\right\}$. We assume that neither the tropical intersection $L_{2}^{3} \cdot H$ contains a classical line nor $C$ is a multiple of $L_{2}^{3} \cdot H$, and show that in this case $C$ is not realizable in $L$. The cases of transversal and non-transversal intersection of $L_{2}^{3}$ with $H$ will in the following be handled separately.

If $L_{2}^{3}$ and $H$ do not intersect transversally, then $H$ contains a maximal cone of $L_{2}^{3}$. Without loss of generality we may assume $H=\left\{[x] \in \mathbb{R}^{4} /\langle\mathbf{1}\rangle: x_{2}=x_{3}\right\}$. Then the geometric intersection is given by

$$
L_{2}^{3} \cap H=\operatorname{cone}\left(\left[e_{0}\right],\left[e_{1}\right]\right) \cup \operatorname{cone}\left(\left[e_{2}+e_{3}\right]\right),
$$

so $P(C)$ has the form $P(C)=\left\{\left(b_{1}, c_{1}, 0,0\right), \ldots,\left(b_{r}, c_{r}, 0,0\right),(0,0, d, d)\right\}$, where $d=\operatorname{deg}(C)$ and $\sum_{k} b_{k}=\sum_{k} c_{k}=d$. Since $C$ was assumed not to be a multiple of the tropical intersection

$$
L_{2}^{3} \cdot H=\operatorname{cone}\left(\left[e_{0}\right]\right) \cup \operatorname{cone}\left(\left[e_{1}\right]\right) \cup \operatorname{cone}\left(\left[e_{2}+e_{3}\right]\right)
$$

(see Lemma 2.11), there is $k \in\{1, \ldots, r\}$ with $a_{k}>0$ and $b_{k}>0$. Let $D_{1} \subset L_{2}^{3}$ be the classical line with $P\left(D_{1}\right)=\{(1,1,0,0),(0,0,1,1)\}$. Then by Construction 2.12 we have $C \cdot D_{1}=d-\sum_{k=1}^{r} \min \left\{a_{k}, b_{k}\right\}-d<0$, and since $C$ does not contain $D_{1}$ by assumption, the tropical curve $C$ is not realizable in $L$ by Proposition 5.10.

We now assume that $L_{2}^{3}$ and $H$ intersect transversally, so that $C$ is contained in $D:=L_{2}^{3} \cdot H$ as a set. By possibly replacing $a$ by $-a$ we may assume that $\left|\left\{i: a_{i}>0\right\}\right| \leq\left|\left\{i: a_{i}<0\right\}\right|$. In particular, we have $\left|\left\{i: a_{i}>0\right\}\right| \in\{1,2\}$. We consider the following two cases:

Case 1: $\left|\left\{i: a_{i}>0\right\}\right|=1$, without loss of generality $a_{0}>0$. By Lemma 2.11 we know that

$$
P(D)=\left\{\left(-a_{1}, a_{0}, 0,0\right),\left(-a_{2}, 0, a_{0}, 0\right),\left(-a_{3}, 0,0, a_{0}\right)\right\} .
$$


But the only balanced curves supported on these three rays are multiples of $D$. So we arrive at a contradiction to our assumption that $C$ is not such a multiple.

Case 2: $\left|\left\{i: a_{i}>0\right\}\right|=2$, and hence, also $\left|\left\{i: a_{i}<0\right\}\right|=2$. By a coordinate permutation and possibly replacing $a$ by $-a$ we can assume that $\left|a_{0}\right|=\max \left\{\left|a_{i}\right|: i=0, \ldots, 3\right\}$ as well as $a_{0}, a_{1}>0$ and $a_{2}, a_{3}<0$. Then Lemma 2.11 tells us that

$$
P(D)=\left\{\left(-a_{2}, 0, a_{0}, 0\right),\left(-a_{3}, 0,0, a_{0}\right),\left(0,-a_{2}, a_{1}, 0\right),\left(0,-a_{3}, 0, a_{1}\right)\right\},
$$

and thus $P(C)$ is given by

$$
P(C)=\left\{\lambda_{02}\left(-a_{2}, 0, a_{0}, 0\right), \lambda_{03}\left(-a_{3}, 0,0, a_{0}\right), \lambda_{12}\left(0,-a_{2}, a_{1}, 0\right), \lambda_{13}\left(0,-a_{3}, 0, a_{1}\right)\right\}
$$

for some $\lambda_{i j} \in \mathbb{Q}_{\geq 0}$, where by abuse of notation we allow some of these coefficients to be zero. Note however that $\lambda_{02}$ cannot be zero: otherwise Lemma 2.9 implies

$$
-\lambda_{03} a_{3}=d=\lambda_{03} a_{0}+\lambda_{13} a_{1}
$$

by adding the 0 -th and last coordinates of these vectors, respectively. This means that $\lambda_{03}\left(a_{0}+a_{3}\right)+\lambda_{13} a_{1}=0$, which is only possible if $\lambda_{13}=0$ as well because $a_{0}+a_{3} \geq 0$ and $a_{1}>0$. But then $C$ consists of only two rays and can thus only be balanced if it is a multiple of a classical line, which we excluded. This contradiction shows that $\lambda_{02}>0$. Of course, by symmetry we then get $\lambda_{03}>0$ as well.

We want to use Proposition 5.20 with $(i, j, k, l)=(0,1,2,3)$ to show that $C$ is not realizable in $L$. So let us check that the assumptions of this proposition are met. It is obvious that $C$ does not intersect cone $\left(\left[e_{0}\right],\left[e_{1}\right]\right)$. We now claim that $C$ meets neither cone $\left(\left[e_{0}\right],\left[e_{0}+e_{2}\right]\right)$ nor cone $\left(\left[e_{0}\right],\left[e_{0}+e_{3}\right]\right)$. This is equivalent to claiming that $a_{0}+a_{2}>0$ and $a_{0}+a_{3}>0$. Assume this is not the case, so without loss of generality we assume $a_{0}+a_{2}=0$. But then $D$ contains the classical line $\operatorname{span}([1,0,1,0])$, which is a contradiction to our assumption.

So, to apply Proposition 5.20, the only thing left to show is that with

$$
c_{1}:=\sum_{a e_{1}+b e_{2} \in P(C)} a=-\lambda_{12} a_{2} \quad \text { and } \quad c_{2}:=\sum_{a e_{0}+b e_{2} \in P(C)} a=-\lambda_{02} a_{2}
$$

we have $c_{1} \neq c_{2}$ and $c_{2} \notin\{0, d\}$. If $c_{1}=c_{2}$ we get $\lambda_{02}=\lambda_{12}$, and hence, by balancing also $\lambda_{02}=\lambda_{03}=\lambda_{12}=\lambda_{13}$. In this case $C$ would be a multiple of $D$, which we excluded. As $\lambda_{02}>0$ we have $c_{2} \neq 0$, and since $d=c_{2}-\lambda_{03} a_{3}$ by Lemma 2.9 and $\lambda_{03}>0$, we also have $c_{2} \neq d$. So $C$ fulfills all the conditions of Proposition 5.20 and thus is not realizable in $L$.

Example 5.22. Proposition 5.20 is more general than the obstruction by Bogart and Katz in [BK11], even in characteristic 0. Consider for instance the tropical curve $C$ of degree 5 in $L_{2}^{3}$ with

$$
P(C)=\{(1,0,2,0),(2,0,3,0),(0,1,0,2),(0,1,0,3),(2,3,0,0)\} .
$$

It does not lie in a classical plane, so the obstruction of Bogart and Katz cannot be applied. But $C$ is not realizable in $L$ by Proposition 5.20 with $(i, j, k, l)=(0,3,1,2)$ if $\operatorname{char}(K)=0$ or $\operatorname{char}(K) \geq 5$ : we have $c_{1}=5, c_{2}=2$, and

$$
C \cap \operatorname{cone}\left(\left[e_{0}\right],\left[e_{3}\right]\right)=C \cap \operatorname{cone}\left(\left[e_{0}\right],\left[e_{0}+e_{1}\right]\right)=C \cap \operatorname{cone}\left(\left[e_{0}\right],\left[e_{0}+e_{2}\right]\right)=\emptyset .
$$


Example 5.23 (Non-realizable curves of small degree). For characteristic 0, the following table contains a complete list, as obtained by Algorithm 4.15, of all tropical curves in $L_{2}^{3}$ (up to symmetry by coordinate permutations) which have degree at most 3 and are not realizable in $L$. To get a feeling of the power of each obstruction presented, we always indicate by which obstruction the non-realizability may be proved.

\begin{tabular}{|c|c|c|c|c|c|}
\hline$P(C)$ & Brugallé-Shaw & 5.10 & 5.15 & 5.17 & 5.20 \\
\hline$\{(2,2,0,0),(0,0,2,1),(0,0,0,1)\}$ & $\checkmark$ & $\checkmark$ & & $\checkmark$ & \\
\hline$\{(3,3,0,0),(0,0,3,2),(0,0,0,1)\}$ & $\checkmark$ & $\checkmark$ & & $\checkmark$ & \\
\hline$\{(3,3,0,0),(0,0,3,1),(0,0,0,2)\}$ & $\checkmark$ & $\checkmark$ & & & \\
\hline$\{(3,3,0,0),(0,0,2,1),(0,0,1,2)\}$ & $\checkmark$ & $\checkmark$ & & & \\
\hline$\{(3,3,0,0),(0,0,2,1),(0,0,1,1),(0,0,0,1)\}$ & $\checkmark$ & $\checkmark$ & & $\checkmark$ & \\
\hline$\{(3,3,0,0),(0,0,2,1),(0,0,1,0),(0,0,0,2)\}$ & $\checkmark$ & $\checkmark$ & & & \\
\hline$\{(3,2,0,0),(0,1,1,0),(0,0,2,2),(0,0,0,1)\}$ & $\checkmark$ & $\checkmark$ & & & \\
\hline$\{(3,2,0,0),(0,1,0,2),(0,0,3,1)\}$ & $\checkmark$ & & & & $\checkmark$ \\
\hline$\{(3,2,0,0),(0,1,0,1),(0,0,3,2)\}$ & $\checkmark$ & $\checkmark$ & & & \\
\hline$\{(3,2,0,0),(0,1,0,1),(0,0,2,1),(0,0,1,1)\}$ & $\checkmark$ & $\checkmark$ & & & \\
\hline$\{(3,2,0,0),(0,1,0,0),(0,0,3,2),(0,0,0,1)\}$ & $\checkmark$ & $\checkmark$ & & & \\
\hline$\{(3,2,0,0),(0,1,0,0),(0,0,2,2),(0,0,1,0),(0,0,0,1)\}$ & $\checkmark$ & $\checkmark$ & & & \\
\hline$\{(3,2,0,0),(0,1,0,0),(0,0,2,1),(0,0,1,2)\}$ & $\checkmark$ & $\checkmark$ & & $\checkmark$ & \\
\hline$\{(3,2,0,0),(0,1,0,0),(0,0,2,1),(0,0,1,1),(0,0,0,1)\}$ & $\checkmark$ & $\checkmark$ & & & \\
\hline$\{(3,1,0,0),(0,1,3,0),(0,1,0,2),(0,0,0,1)\}$ & & & $\checkmark$ & & \\
\hline$\{(2,2,0,0),(1,0,0,0),(0,1,0,0),(0,0,2,1),(0,0,1,2)\}$ & $\checkmark$ & $\checkmark$ & & & \\
\hline$\{(2,1,0,0),(1,2,0,0),(0,0,2,1),(0,0,1,2)\}$ & $\checkmark$ & $\checkmark$ & & & \\
\hline$\{(2,1,0,0),(1,2,0,0),(0,0,2,1),(0,0,1,1),(0,0,0,1)\}$ & $\checkmark$ & $\checkmark$ & & $\checkmark$ & \\
\hline
\end{tabular}

In the list above, we indicated that the non-realizability of the tropical curve $C$ with $P(C)=$ $\{(3,2,0,0),(0,1,0,2),(0,0,3,1)\}$ can be proved using the obstruction by Brugallé-Shaw. This results from the fact that $C \cdot D<0$, where $D$ is the tropical curve in $L_{2}^{3}$ with $P(D)=$ $\{(6,4,0,0),(0,2,5,0),(0,0,1,3),(0,0,0,3)\}$. Using for instance Algorithm 4.15, one sees that $D$ is realizable in $L$.

Contrary to this observation, one can show that if $C$ is the tropical curve in $L_{2}^{3}$ with $P(C)=$ $\{(3,1,0,0),(0,1,3,0),(0,1,0,2),(0,0,0,1)\}$, then $C \cdot D \geq 0$ for all tropical curves $D$ in $L_{2}^{3}$. Hence, the obstruction by Brugallé-Shaw cannot be used to prove the non-realizability of C.

In degree 4 there are 138 tropical curves (up to coordinate permutations) in $L_{2}^{3}$ which are not realizable in $L$ if $\operatorname{char}(K)=0$. We see in the list above that Proposition 5.10 is a strong obstruction. In fact, among these 138 non-realizable degree- 4 curves in $L_{2}^{3}$ there are only 22 curves whose non-realizability cannot be proved using Proposition 5.10. We are now listing these 22 curves with indications which obstruction can be used to prove the nonrealizability.

\begin{tabular}{||c||c|c|c||}
$P(C)$ & 5.15 & 5.17 & 5.20 \\
\hline$\{(4,3,0,0),(0,1,0,3),(0,0,4,1)\}$ & & & $\checkmark$ \\
$\{(4,3,0,0),(0,1,0,3),(0,0,3,1),(0,0,1,0)\}$ & & & $\checkmark$
\end{tabular}



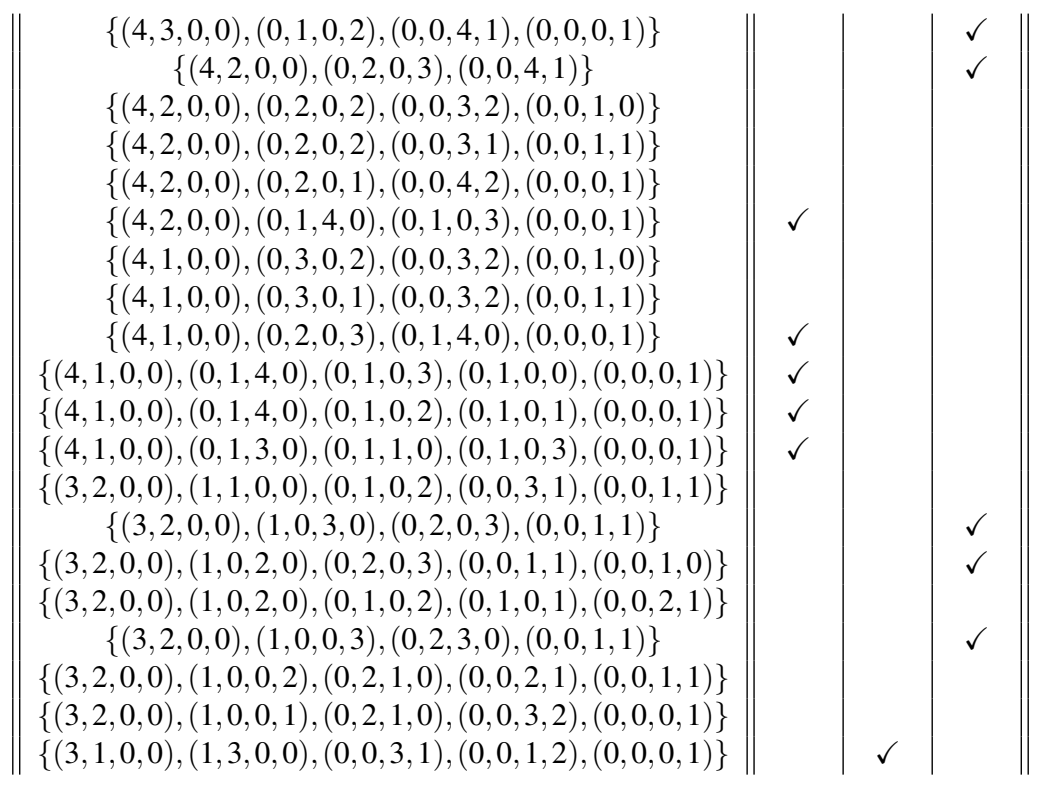

Example 5.24 (Realizability depends on $\operatorname{char}(K)$ ). Some of our criteria for realizability in this section had a dependence on the characteristic of $K$. The following example shows that the relative realizability of a tropical curve in $L_{2}^{3}$ may in fact depend on $\operatorname{char}(K)$ : consider the curves $C, D$ in $L_{2}^{3}$ with

$$
\begin{aligned}
P(C) & =\{(0,0,3,1),(0,1,0,2),(3,2,0,0)\}, \\
P(D) & =\{(0,0,1,0),(0,0,2,1),(0,1,0,2),(3,2,0,0)\} .
\end{aligned}
$$

For $\operatorname{char}(K)=0$ we see in the list above that $C$ is not realizable in $L$, but $D$ is. However, using our algorithm we get for $\operatorname{char}(K)=2$ that $C$ is realizable in $L$, while $D$ is not. Hence, the realizability in $L$ depends on the characteristic of $K$.

\section{REFERENCES}

[AK06] Federico Ardila and Caroline Klivans, The bergman complex of a matroid and phylogenetic trees, J. Comb. Theory, Ser. B 96 (2006), no. 1, 38-49, arXiv: 0311370v2.

[AR10] Lars Allermann and Johannes Rau, First steps in tropical intersection theory, Math. Z. 264 (2010), no. 3, 633-670, arXiv: 0709.3705.

[BG86] Robert Bieri and John Groves, A rigidity property for the set of all characters induced by valuations, Trans. Amer. Math. Soc. 294 (1986), 425-434.

[BK11] Tristam Bogart and Eric Katz, Obstruction to lifting tropical curves in surfaces in 3-space, arXiv: 1101.0297, 2011.

[BS11] Erwan Brugallé and Kristin Shaw, Obstructions to approximating tropical curves in surfaces via intersection theory, arXiv: 1110.0533, 2011.

[DGPS] Wolfram Decker, Gert-Martin Greuel, Gerhard Pfister, and Hans Schönemann, Singular - a computer algebra system for polynomial computations, http://www.singular.uni-kl.de.

[Eis95] David Eisenbud, Commutative algebra with a view towards algebraic geometry, Springer, 1995. 
[EKL06] Manfred Einsiedler, Mikhail Kapranov, and Douglas Lind, Non-archimedean amoebas and tropical varieties, J. Reine Angew. Math. 601 (2006), 139-157.

[FR10] Georges François and Johannes Rau, The diagonal of tropical matroid varieties and cycle intersections, arXiv: 1012.3260, 2010.

[Fra12] Georges François, Tropical intersection products and families of tropical curves, Ph.D. thesis, University of Kaiserslautern, 2012, urn:nbn:de:hbz:386-kluedo-33502.

[FS97] William Fulton and Bernd Sturmfels, Intersection theory on toric varieties, Topology 36 (1997), no. 2, 335-353, arXiv: alg-geom/9403002.

[Fu198] William Fulton, Intersection theory, Springer, 1998.

[GKM09] Andreas Gathmann, Michael Kerber, and Hannah Markwig, Tropical fans and the moduli space of rational tropical curves, Compos. Math. 145 (2009), no. 1, 173-195, arXiv: 0708.2268.

[Har77] Robin Hartshorne, Algebraic geometry, Springer, 1977.

[Mik02] Grigory Mikhalkin, Decomposition into pairs-of-pants for complex algebraic hypersurfaces, arXiv: math/0205011, 2002.

[NS06] Takeo Nishinou and Bernd Siebert, Toric degenerations of toric varieties and tropical curves, Duke Math. J. 135 (2006), 1-51, arXiv:math.AG/0409060.

[Ox192] James Oxley, Matroid theory, Oxford University Press, 1992.

[Rau08] Johannes Rau, Intersections on tropical moduli spaces, arXiv: 0812.3678, 2008.

[Rau09] , Tropical intersection theory and gravitational descendants, Ph.D. thesis, University of Kaiserslautern, 2009, urn:nbn:de:hbz:386-kluedo-23706.

[Sha10] Kristin Shaw, A tropical intersection product in matroidal fans, arXiv: 1010.3967, 2010.

[Spe05] David Speyer, Tropical geometry, Ph.D. thesis, University of California, Berkeley, 2005.

[Spe07] _ Uniformizing tropical curves I: Genus zero and one, arXiv: 0711.2677, 2007.

[ST08] Bernd Sturmfels and Jenia Tevelev, Elimination theory for tropical varieties, Math. Res. Lett. 15 (2008), no. 3, 543-562, arXiv: 0704.3471.

[Stu02] Bernd Sturmfels, Solving systems of linear equations, CBMS Regional Conference Series in Mathematics 97, American Mathematical Society, 2002.

[Win12] Anna Lena Winstel, realizationMatroids.lib - a Singular library for relative realizability questions on tropical curves, http://www.mathematik.uni-kl.de/"gathmann/matroids.php, 2012.

[Zie02] Günter M. Ziegler, Lectures on polytopes, Springer-Verlag New York, 2002.

Andreas Gathmann, Fachbereich Mathematik, Technische Universität Kaiserslautern, PostFach 3049, 67653 Kaiserslautern, GeRmany

E-mail address: andreas@mathematik.uni-kl.de

Kirsten Schmitz, Fachbereich Mathematik, Technische Universität Kaiserslautern, PostFACH 3049, 67653 KAISERSLAUTERN, GERMANY

E-mail address: schmitz@mathematik.uni-kl.de

Anna Lena Winstel, Fachbereich Mathematik, Technische Universität Kaiserslautern, POSTFACH 3049, 67653 KAisERSLAUTERn, GERMANY

E-mail address: winstel@mathematik.uni-kl.de 